\title{
Starburst galaxies in the COSMOS field: clumpy star-formation at redshift $0<z<0.5^{\star}$
}

\author{
R. Hinojosa-Goñi ${ }^{1,2}$, C. Muñoz-Tuñón ${ }^{1,2}$, and J. Méndez-Abreu ${ }^{3}$ \\ 1 Instituto de Astrofísica de Canarias, Calle Vía Láctea s/n, 38200 La Laguna, Tenerife, Spain \\ e-mail: hinojosa@iac.es \\ 2 Departamento de Astrofísica, Universidad de La Laguna, 38205 La Laguna, Tenerife, Spain \\ 3 School of Physics and Astronomy, University of St Andrews, SUPA, North Haugh, KY16 9SS, St Andrews, UK
}

Received 27 July 2015 / Accepted 15 March 2016

\begin{abstract}
Context. At high redshift, starburst galaxies present irregular morphologies with 10-20\% of their star formation occurring in giant clumps. These clumpy galaxies are considered the progenitors of local disk galaxies. To understand the properties of starbursts at intermediate and low redshift, it is fundamental to track their evolution and the possible link with the systems at higher $z$.

Aims. We present an extensive, systematic, and multiband search and analysis of the starburst galaxies at redshift $(0<z<0.5)$ in the COSMOS field, as well as detailed characteristics of their star-forming clumps by using Hubble Space Telescope/Advance Camera for Surveys (HST/ACS) images.

Methods. The starburst galaxies are identified using a tailor-made intermediate-band color excess selection, tracing the simultaneous presence of $\mathrm{H} \alpha$ and $[\mathrm{OIII}]$ emission lines in the galaxies. Our methodology uses previous information from the zCOSMOS spectral database to calibrate the color excess as a function of the equivalent width of both spectral lines. This technique allows us to identify 220 starburst galaxies at redshift $0<z<0.5$ using the SUBARU intermediate-band filters. Combining the high spatial resolution images from the HST/ACS with ground-based multi-wavelength photometry, we identify and parametrize the star-forming clumps in every galaxy. Their principal properties, sizes, masses, and star formation rates are provided.

Results. The mass distribution of the starburst galaxies is remarkably similar to that of the whole galaxy sample with a peak around $M / M_{\odot} \sim 2 \times 10^{8}$ and only a few galaxies with $M / M_{\odot}>10^{10}$. We classify galaxies into three main types, depending on their HST morphology: single knot (Sknot), single star-forming knot plus diffuse light (Sknot+diffuse), and multiple star-forming knots (Mknots/clumpy) galaxy. We found a fraction of Mknots/clumpy galaxy $f_{\text {clumpy }}=0.24$ considering out total sample of starburst galaxies up to $z \sim 0.5$. The individual star-forming knots in our sample follows the same $L(\mathrm{H} \alpha)$ vs. size scaling relation as local giant HII regions. However, they slightly differ from the one provided using samples at high redshift. This result highlights the importance of spatially resolving the star-forming regions for this kind of study. Star-forming clumps in the central regions of Mknots galaxies are more massive, and present higher star formation rates, than those in the outskirts. This trend is less clear when we consider either the mass surface density or surface star formation rate. Sknot galaxies do show properties similar to both dwarf elliptical and irregulars in the surface brightness $(\mu)$ versus $M_{\text {host }}$ diagram in the $B$-band, and to spheroidals and ellipticals in the $\mu$ versus $M_{\text {host }}$ diagram in the $V$-band.

Conclusions. The properties of our star-forming knots in Sknot+diffuse and Mknots/clumpy galaxies support the predictions of recent numerical simulations claiming that they have been produced by violent disk instabilities. We suggest that the evolution of these knots means that large and massive clumps at the galaxy centers represent the end product of the coalescence of surviving smaller clumps from the outskirts. Our results support this mechanism and make it unlikely that mergers are the reason behind the observed starburst knots. Sknot galaxies might be transitional phases of the Blue Compact Dwarfs (BCD) class, with their properties consistent with spheroidal-like, but blue structures.
\end{abstract}

Key words. galaxies: starburst - galaxies: star formation - galaxies: bulges - galaxies: evolution - galaxies: formation galaxies: structure

\section{Introduction}

Understanding the physical processes leading to the formation and evolution of galaxies represents a major question for modern astronomy. Among them, the processes governing the star formation (SF) over cosmic time play a fundamental role in galaxy evolution. At high redshift, the SF in galaxies is mainly fueled by accretion of pristine gas from the cosmic web (Kereš et al. 2005; Dekel et al. 2009; Aumer et al. 2010). In this scenario, when the

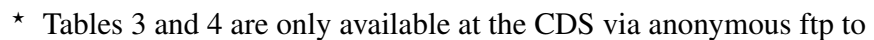
cdsarc.u-strasbg.fr (130.79.128.5) or via

http://cdsarc.u-strasbg.fr/viz-bin/qcat?J/A+A/592/A122
}

dark matter halo is diffuse enough, the cool gas stream from the cosmic web can reach the inner halo, or disk, directly providing fresh gas to form stars. This mechanism is called cold-flow accretion and it is predicted to be the main mode to trigger the SF in the early Universe (L'Huillier et al. 2012). Recently, it has been proven that the accretion of metal-poor gas from the cosmic web may also activate the SF in the disk of nearby galaxies (Sánchez Almeida et al. 2013, 2014). Another scenario used to explain the growth of galaxies at high redshift invokes galaxy mergers (Conselice et al. 2003; Bell et al. 2006; Lotz et al. 2006; Bournaud et al. 2009). In this case, numerical simulations predict that major mergers can contribute up to $20 \%$ of the galaxy 
mass growth (Wang et al. 2011) but at the cost of destroying the galactic disks of their progenitor (Naab et al. 2006; Guo 2011). Therefore, several works suggest that merger-driven starbursts are less important than those triggered by gas accretion from the cosmic web (van de Voort et al. 2011).

Recent observations and numerical simulations agree, showing that the SF at high redshift occurs mainly in giant clumps (Bournaud 2016). The formation mechanism of these SF clumps is still a matter of debate. One scenario proposes that they are formed by disk fragmentation in gravitationally unstable disks (Noguchi 1999; Immeli et al. 2004a,b; Bournaud et al. 2007; Elmegreen et al. 2008). In this case, an intense inflow of cool gas is necessary to provide the high gas surface densities leading to the disk instabilities (Dekel et al. 2009). Contrary to this in situ clump formation, Mandelker et al. (2014) proposed that a limited number of ex situ clumps might also be accreted by minor mergers into the galaxy disk.

The Hubble Deep Field (HDF) and Ultra Deep Field (UDF) have been crucial for characterizing the different morphologies of galaxies at high redshift. Observationally, galaxies at high redshift observed in the HDF or UDF with the Hubble Space Telescope (HST) show clumpy structures with high star formation rates (SFR), which are rare in the local Universe (Abraham et al. 1996; van den Bergh et al. 1996; Elmegreen et al. 2004a,b, 2007). The shape and distribution of the SF knots led Elmegreen et al. (2007) to propose a classification of galaxies as: chains, doubles, tadpoles, clump-clusters, spirals, and ellipticals. Elmegreen et al. (2004a,b) compared the properties of the clumps present in chain galaxies with those of clump-cluster galaxies. They found similar properties, indicating that both are the same kind of galaxies but seen from different line of sights. Genzel et al. (2011) studied the properties of five clumpy galaxies at $z \sim 2$ with deep SINFONI AO spectroscopy. They suggest that the spatial variation of the inferred gas-phase oxygen abundance is broadly consistent with an inside-out growing disk, and/or with inward migration of the clumps. Several studies have confirmed the young nature of the stellar populations in the SF clumps (Elmegreen et al. 2009; Wuyts et al. 2012), with only some rare examples having older stellar populations (Bournaud et al. 2008). At low redshift, SF occurs in a large variety of physical scales. Recently, Elmegreen et al. (2013) made use of the Kiso survey of galaxies (Miyauchi-Isobe et al. 2010) to compare their SF regions with the giant clumps found at high redshift. They found that local clumpy galaxies in the Kiso survey appear to be intermediate between high-redshift clumps and those in normal spirals. Evidence for SF sustained by tadpole, extremely metal-poor (XMP) galaxies via cold gas accretion at low redshift has been shown by Sánchez Almeida et al. (2014).

Numerical simulations predict that, for a given mass, the galaxies become more clumpy at high redshift (Ceverino et al. 2010). The observations by Elmegreen et al. (2007), including galaxies of different masses and at different redshifts, confirm this tendency. These SF clumps are important, not only regarding the mass growth of the galaxy, but also for its morphological evolution. Numerical simulations show that SF clumps can migrate from the outer disk to the galaxy center, and contribute to the formation of bulges. The coalescence of clumps can take place in timescales of $\sim 4$ rotation times $(0.5-0.7 \mathrm{Gyr})$, therefore representing an alternative path for bulge formation at high redshift (Noguchi 1999; Immeli et al. 2004a; Bournaud et al. 2007; Ceverino et al. 2010). The pre-existing thick disk and the SF clump migration to the center have also been considered as responsible for the exponential profile in the luminosity of the present spiral galaxies (Bournaud et al. 2007; Elmegreen et al. 2013). As a result of this evolution, the system transforms from an initially uniform disk with high mass SF clumps to spiral-like galaxies with an exponential or double-exponential disk profile, a central bulge, and small remaining clumps (Bournaud et al. 2007; Ceverino et al. 2010; Elmegreen et al. 2013).

Following the predictions of numerical simulations, observational studies of clumpy galaxies have been performed using mainly high-redshift samples $(z>1)$. Deep, high-redshift surveys have provided clues for studying the evolution of disk galaxies, with investigations spanning from the characterization and precise study of the SF knots (clumps), to their relation to the host galaxies. However, these high-redshift studies are usually severely limited by the spatial resolution of the images, even when using space-based observations with the HST Advanced Camera for Surveys (ACS). At lower redshift, studies have been mostly restricted to spectroscopic samples (Amorín et al. 2012). We aim to bridge the gap between high-redshift galaxy studies and those in the local Universe by providing an accurate characterization of starburst galaxies at intermediate redshift, to constrain models of disk galaxy formation. In the present work we analyze a photometrically selected complete sample of starburst galaxies at redshift $0<z<0.5$. The analysis and catalogue of the targets at redshift $z>0.5$ will be the topic of a forthcoming publication.

The structure of this paper is as follows: in Sect. 2, we describe the databases used and our methodology for identifying starburst galaxies in the COSMOS field. In Sect. 3, we determine the $K$-correction and stellar masses of our sample galaxies. In Sect. 4 we analyze the morphology of the galaxies, and derive the properties of the starburst knots using the HST images. Section 5 presents the properties of the galaxies and star-forming regions. The comparison with high-redshift galaxies is also shown. In Sect. 6 we provide conclusions. A standard $\Lambda$ cold dark matter cosmology with $\Omega_{\mathrm{m}}=0.27, \Omega_{\Lambda}=0.71$, and $H_{0}=0.7$ is adopted throughout this paper.

\section{COSMOS-selected starburst sample}

COSMOS is the largest deep field survey ever done by the HST. It covers two equatorial square degrees, and about $2 \times 10^{9}$ galaxies have been observed in this area with the ACS. Space and ground telescopes have been used to map a wide range of the electromagnetic spectrum, from radio waves to X-ray. In this work we use the photometric redshift catalogue (Ilbert et al. 2008), the COSMOS intermediate and broadband photometric catalogue (Capak et al. 2007) and the spectra available in $z$ COSMOS (Lilly et al. 2007), to search for starburst galaxies.

\section{COSMOS intermediate and broadband photometry catalogue}

Over 2 million sources have been observed with the HST/ACS high spatial resolution camera, using the $F 814 \mathrm{~W}$ filter in the COSMOS field. Additional observations in 30 bands, covering the ultraviolet (UV) to the infrared (IR), were made using different ground- and space-based instruments, and are available in this catalogue. To perform our search we used the observations with the intermediate-band filters from the SUBARU telescope.

\section{COSMOS photometric redshift catalogue}

To take spectra of thousands of sources is very time-consuming. Therefore, measuring redshifts based only on photometric data is more efficient and has been demonstrated to be a powerful 
tool in recent surveys (Ilbert et al. 2009; Benítez et al. 2009). The photometric redshift $\left(z_{\text {phot }}\right)$ in COSMOS was computed using 30 broad, intermediate, and narrow-band filters covering the UV, visible-near infrared (NIR), and mid-IR spectral ranges (Ilbert et al. 2009). This catalogue contains 385065 sources classified as galaxies, stars, $\mathrm{X}$-ray sources, faint sources, or masked areas. Among them, we choose only the 305002 sources flagged as galaxies. To measure the $\mathrm{H} \alpha$ and [OIII] emission lines, we used the SUBARU filters and the $z_{\text {phot }}$. To this aim, we matched the COSMOS intermediate and broadband photometry catalogue with the COSMOS photometric redshift catalogue.

\section{zCOSMOS}

The spectroscopic redshift $(z)$ was obtained from observations of the VIsible MultiObject Spectrograph (VIMOS) on the Very Large Telescope (VLT) for a subsample of the COSMOS field, as part of the $z$ COSMOS project (Lilly et al. 2007). This catalogue contains two parts. The first is $z$ COSMOS-bright, aimed at observing $\sim 20000$ galaxies at $0.1<z<1.2$ using the VIMOS red spectral range (5550-9650 $\mathrm{A}$ ) with the $R \sim 600 \mathrm{MR}$ grism, to detect the strong spectral features around $4000 \AA$. The second part is the $z$ COSMOS-deep, which aims at observing $\sim 10000$ galaxies lying at $1.5<z<2.5$ in the blue spectral range (3600-6800 $\AA$ ), to measure the strong absorption and emission features in the range between 1200 and $1700 \AA$. At the moment, there are 10643 published spectra, corresponding to the results of the $z$ COSMOS-bright spectroscopic observations, which were carried out in VLT Service Mode during the period April 2005 to June 2006. These have been observed using a 1 arcsec-wide slit, sampling roughly $2.5 \AA /$ pixel, with a velocity accuracy of $\sim 100 \mathrm{~km} \mathrm{~s}^{-1}$.

\subsection{Starbursts in zCOSMOS}

Starburst galaxies show a steep rising continuum in the blue region of the spectra, combined with strong nebular emission lines. At visible wavelengths, the [OIII] and $\mathrm{H} \alpha$ emission lines are the most prominent and we can parametrize them using the equivalent width (EW). Previous studies of starburst galaxies in the local Universe (Kniazev et al. 2004; Cairós et al. 2007, 2009a,b, 2010; Morales-Luis et al. 2011; Amorín et al. 2014) found minimum values of the [OIII] and $\mathrm{H} \alpha \mathrm{EW}$ of about $80 \AA$. This value corresponds to young star-forming regions, with ages $<10 \mathrm{Myr}$ (Leitherer et al. 1999, 2010; Vázquez \& Leitherer 2005). Therefore, the EW turns out to be the best parameter to identify young starburst galaxies and we adopt the aforementioned EW threshold values in our work.

To measure the EW of the [OIII] and $\mathrm{H} \alpha$ emission lines, we first used the spectra from $z$ COSMOS. The wavelength range of the spectra allows us to simultaneously measure [OIII] and $\mathrm{H} \alpha$ in the redshift range $0.1 \leq z \leq 0.47$. zCOSMOS comprises 3384 galaxies in this redshift range. To identify those with emission lines, we used the published spectroscopic redshift and the rest-frame wavelengths of the [OIII] and $\mathrm{H} \alpha$ emission lines $\left(\lambda_{\mathrm{c}}\right.$ : $4959,5007 \AA ; 6563 \AA$ ). Then, we measure the flux of the emission line $\left(F_{l}\right)$ in a baseline of $30 \AA$, which allows us to cover the total width of the line at the continuum level. The same bandwidth was used to measure the flux of the continua $\left(F_{\mathrm{c}}\right)$ at restframe wavelengths $5053 \AA$ and $6518 \AA$ for [OIII] and $\mathrm{H} \alpha$, respectively. The EW is defined as

$E W=\sum \frac{F_{1}-F_{\mathrm{c}}}{F_{\mathrm{c}}} \delta \lambda$,
Table 1. Starburst galaxies selected from zCOSMOS.

\begin{tabular}{cccc}
\hline \hline Object & $z$ & $\begin{array}{c}E W(\mathrm{H} \alpha) \\
(\AA)\end{array}$ & $\begin{array}{c}E W([\mathrm{OIII}]) \\
(\AA)\end{array}$ \\
$(1)$ & $(2)$ & $(3)$ & $(4)$ \\
\hline cosmos-002 & 0.34 & $234 \pm 43$ & $321 \pm 18$ \\
cosmos-003 & 0.25 & $104 \pm 14$ & $108 \pm 28$ \\
cosmos-010 & 0.13 & $144 \pm 19$ & $100 \pm 11$ \\
cosmos-014 & 0.38 & $208 \pm 21$ & $124 \pm 7$ \\
cosmos-015 & 0.41 & $94 \pm 21$ & $166 \pm 16$ \\
cosmos-017 & 0.17 & $177 \pm 58$ & $121 \pm 12$ \\
cosmos-018 & 0.28 & $193 \pm 40$ & $174 \pm 10$ \\
cosmos-021 & 0.19 & $96 \pm 19$ & $126 \pm 8$ \\
cosmos-026 & 0.44 & $148 \pm 70$ & $115 \pm 18$ \\
cosmos-027 & 0.34 & $190 \pm 31$ & $164 \pm 6$ \\
\hline
\end{tabular}

Notes. (1) Object name (ordered by RA); (2) spectroscopic redshift; (3) $\mathrm{H} \alpha$ EW; and (4) [OIII] EW.

where $\delta \lambda=2.5 \AA$. The associated error is given by

$e_{\mathrm{EW}}=\frac{F_{1}}{F_{\mathrm{c}}}\left(\frac{\sigma_{\mathrm{c}}}{\operatorname{med}_{\mathrm{c}}} \cdot \sqrt{8 n}\right)$

where both $\sigma_{\mathrm{c}}$ and $\operatorname{med}_{\mathrm{c}}$ correspond to the standard deviation and the median in the continuum, respectively, and $n$ is the number of resolution elements in the selected baseline. We calculated the EW in $\mathrm{H} \alpha$ and [OIII] for all 3384 galaxies, covering the range $0.1 \leq z \leq 0.47$. We selected those with $E W \geq 80 \AA$ in $\mathrm{H} \alpha$ and [OIII], obtaining a sample of 82 starburst galaxies. Table 1 shows the first few entries of the spectroscopic emission-line catalogue. The complete catalogue is available in Table 3.

\subsection{Starbursts in the COSMOS photometric catalogue}

Only $\sim 2.8 \%$ of objects in COSMOS have a spectroscopic redshift. Therefore, the use of the photometric redshift is fundamental for producing a complete catalogue of starburst galaxies. In the following, we describe the color-color selection method used to detect galaxies with $E W \geq 80 \AA$ in both $\mathrm{H} \alpha$ and [OIII]. We also show how we calibrate the method using the available spectral information.

\subsubsection{Calibration of color-color diagnostics with spectra}

Color-color diagrams using narrow/intermediate bands have been successfully used in the past to select samples of emission line-galaxies (Sobral et al. 2013). However, the definition of the boundaries between different galaxy types, generally starforming versus passive, is often subjective and dependent on the characteristics of the objects under study.

To calibrate our color-color diagnostic diagram, we used the sample of confirmed starburst galaxies in the spectroscopic catalogue (see Sect. 2.1) as templates to search for a complete sample in the COSMOS photometric sample. These galaxies were selected as starbursts based on their EW in both $\mathrm{H} \alpha$ and [OIII]. We used the spectroscopic redshifts to locate the emission lines in the SUBARU intermediate band filters. We also selected two regions free of lines for the continuum, centered at $5500 \AA$ (C5500) as continuum for the [OIII] emission line, and at $6000 \AA$ (C6000) for the $\mathrm{H} \alpha$ emission line. With these we constructed color-color diagrams, matching the presence of the corresponding strong line and its continuum. The colors constructed 


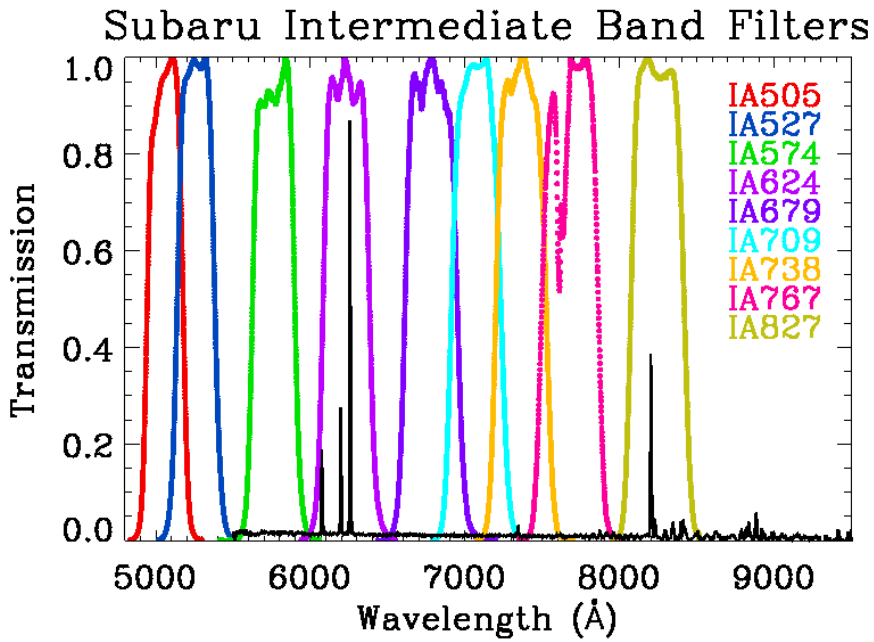

Fig. 1. Normalized transmission curves of the intermediate-band SUBARU filters used in this study. A representative emission-line galaxy at $z=0.25$ is also shown to demonstrate how the filters match simultaneously the positions of the [OIII] and $\mathrm{H} \alpha$ lines. The space between filters determine the wavelength gaps in our photometric sample.

are SUBARU(H $\alpha)$ - SUBARU(C6000) vs. SUBARU([OIII]) SUBARU(C5500). Figure 1 shows the transmission of the SUBARU filters, with an example starburst galaxy at redshift 0.25. We note that SUBARU filters do not homogeneously cover the whole range; instead some gaps can clearly be seen in Fig. 1. The redshift range where we are able to locate simultaneously $\mathrm{H} \alpha$ and [OIII] lines are: $0.123<z<0.178$ and $0.23<$ $z<0.274$. The total number of galaxies in $z$ COSMOS within these redshifts are 580, of which 24 are starburst galaxies. Their color-color diagram (SUBARU(H $\alpha$ ) - SUBARU(C6000) versus SUBARU([OIII]) - SUBARU(C5500)) is shown in Fig. 2a. Galaxies with $E W \geq 80 \AA$ in both $\mathrm{H} \alpha$ and [OIII], measured from the spectra, are represented by stars. The arrow in Fig. 2a shows the direction of increasing emission-line EW. As expected, starburst galaxies populate a well-defined region of the color-color diagram. The use of galaxies with spectra enables us to calibrate the color excess as a function of the emission-line EWs and therefore use the easily accessible photometric data to select starburst galaxies with $E W \geq 80 \AA$ in both $\mathrm{H} \alpha$ and [OIII].

We use the region where $\mathrm{H} \alpha-\mathrm{C} 6000 \leq-0.35$ and [OIII]$\mathrm{C} 5500 \leq-0.1$ (shadow region in Fig. 2a) as the proxy for emission associated with starburst galaxies. This region is used to search for starburst candidates using the photometric redshift catalogue in the next section.

\subsubsection{Starbursts using the COSMOS photometric redshift catalogue}

The photometric redshift allows us to match the $\mathrm{H} \alpha$ and [OIII] emission lines in the SUBARU intermediate-band filters in the redshift ranges: $0.007 \leq z \leq 0.074,0.124 \leq z \leq 0.177$, and $0.230 \leq z \leq 0.274$. The bands, including [OIII], $\mathrm{H} \alpha$, and their respective continuum at $5500 \AA$ and $6000 \AA$, have been used to build a color-color diagnostic diagram (shown in Fig. 2b) similar to the one for the spectroscopic sample. We limit our sample to galaxies with $m(F 814 W)<23.5$. Fainter galaxies have both photometric redshift and intermediate-band magnitude errors that are too large for our analysis. To estimate the photometric EW, we used a filter in the red continuum for [OIII] and
Table 2. Starburst galaxies selected from the photometric catalogue.

\begin{tabular}{cccc}
\hline \hline Object & $z_{\text {phot }}$ & $\begin{array}{c}E W(\mathrm{H} \alpha) \\
(\AA)\end{array}$ & $\begin{array}{c}E W([\mathrm{OIII}]) \\
(\AA)\end{array}$ \\
$(1)$ & $(2)$ & $(3)$ & $(4)$ \\
\hline cosmos-001 & 0.27 & $760 \pm 46$ & $770 \pm 43$ \\
cosmos-004 & 0.26 & $119 \pm 26$ & $171 \pm 26$ \\
cosmos-005 & 0.26 & $94 \pm 10$ & $182 \pm 11$ \\
cosmos-006 & 0.26 & $124 \pm 11$ & $125 \pm 10$ \\
cosmos-007 & 0.26 & $108 \pm 19$ & $112 \pm 16$ \\
cosmos-008 & 0.16 & $110 \pm 34$ & $60 \pm 30$ \\
cosmos-009 & 0.06 & $28 \pm 10$ & $109 \pm 11$ \\
cosmos-011 & 0.26 & $351 \pm 13$ & $568 \pm 16$ \\
cosmos-012 & 0.01 & $114 \pm 7$ & $55 \pm 5$ \\
cosmos-013 & 0.26 & $114 \pm 27$ & $181 \pm 26$ \\
\hline
\end{tabular}

Notes. (1) Object name (ordered by RA); (2) photometric redshift; (3) $\mathrm{H} \alpha$ EW; and (4) [OIII] EW.

in the blue continuum for $\mathrm{H} \alpha$. Then we used Eq. (1) to calculate the EW for both emission lines. In Fig. 2b, we plot in the color-color diagram defined using the SUBARU filters for all the galaxies in the $z$ COSMOS catalogue. The star symbols show the locii for the starburst galaxies within the region with $E W \geq 80 \AA$.

In Fig. 2b, a smaller region is shown with a darker shadow, with $\mathrm{H} \alpha$-C6000 $\leq-0.5$ and [OIII]-C5500 $\leq-0.4$. In the larger region, which we used to obtain our sample, only $33 \%$ of the photometric EW in $\mathrm{H} \alpha$ or [OIII] are less than $80 \AA$. The smaller region exclusively comprises objects where the photometric EW in both $\mathrm{H} \alpha$ and [OIII] is $\geq 80 \AA$. These are the targets included in the catalogue that is presented in this work. Table 2 shows some of the entries of the photometric emission-line catalogue, constructed using only the galaxies in the darker shadowed area (star symbols in Fig. 2b). The complete catalogue is available in Table 3.

\subsection{Caveats to the sample selection}

Several comparisons have been made to assure that our photometric sample is reliable, and to establish possible sources of uncertainty. We have detected inconsistencies in some of the photometric redshift estimations. To have a secure sample, other criteria have been used to filter our sample; galaxies with $\mathrm{H} \alpha$ emission-line only (without [OIII]) have been identified in Fig. 2b (see horizontal branch with $0<$ [OIII]-C5500 $<-0.5$ ) and discarded. Comparing photometric and spectroscopic redshifts, we detected some inconsistencies in the photometric redshift estimation for $z \leq 0.1$. As the colors shown in the diagram use the photometric redshift, the diagram is also useful for detecting inconsistencies in $z_{\text {phot }}$ for emission-line galaxies. For these galaxies at $z \leq 0.1$, the photometric redshift confused [OII] with [OIII], and [OIII] with $\mathrm{H} \alpha$ emission lines. This problem, known as "catastrophic redshift", was reported in Ilbert et al. (2008, their Fig. 1). In summary, since we look for starburst galaxies showing simultaneously $\mathrm{H} \alpha$ and [OIII], when discarding galaxies in the color-color diagram with high $\mathrm{H} \alpha$ and low [OIII], we automatically remove galaxies with wrong photometric redshift determinations.

Altogether, we have identified a total number of 289 starburst galaxies with $E W \geq 80 \AA$ in $\mathrm{H} \alpha$ and [OIII] in COSMOS, either from the photometric or spectroscopic catalogues. From 


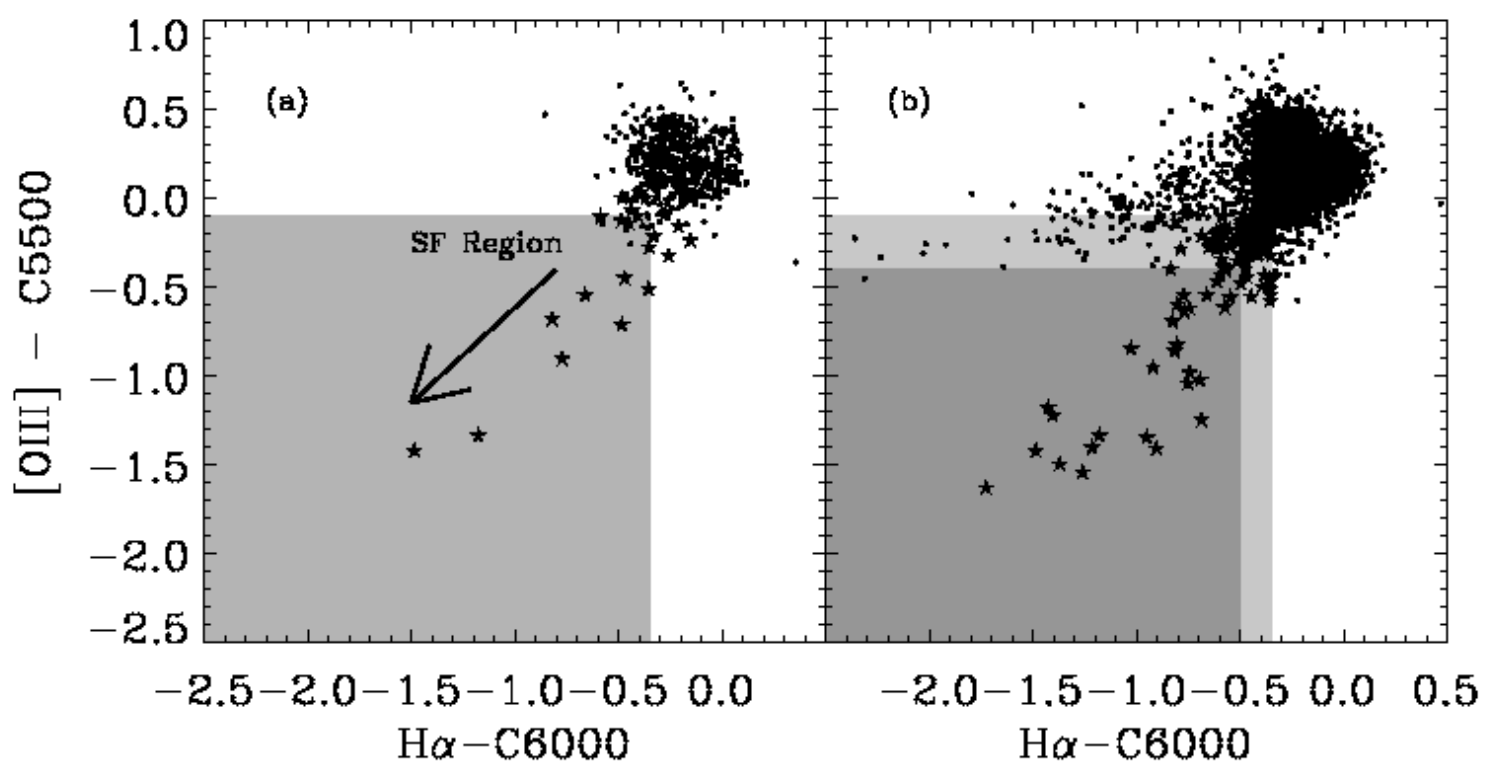

Fig. 2. a) Color-color diagram of the 580 galaxies in $z$ COSMOS measured with SUBARU filters. Star e show the galaxies with $E W \geq 80 \AA$ in both $\mathrm{H} \alpha$ and [OIII]. The arrow points in the direction of galaxies with larger EW in both emission lines. The shadowed region in the diagram is the starburst location. b) Color-color diagram for galaxies in the photometric redshift catalogue, in the redshift ranges: $0.007 \leq z \leq 0.074$, $0.124 \leq z \leq 0.177$, and $0.230 \leq z \leq 0.274$. Star symbols show the galaxies in the star-forming region defined in panel a). We discarded galaxies with high $\mathrm{H} \alpha$ and low [OIII] emission. A subregion (darker shadow) shows the loci in which the photometric $\mathrm{EW}$ in $\mathrm{H} \alpha$ and [OIII] is larger than $80 \AA$ for the photometric sample.
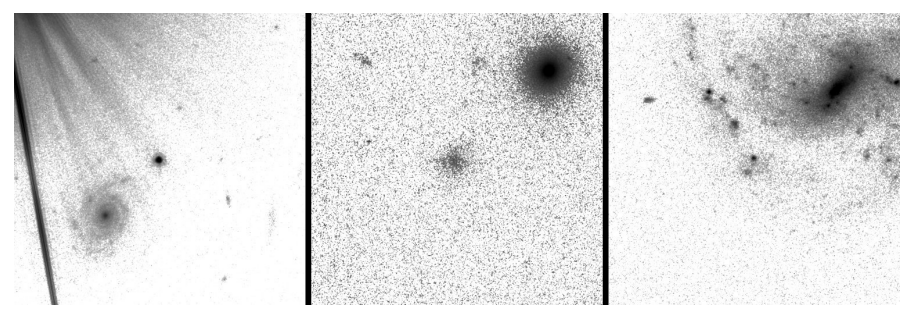

Fig. 3. HST images with some examples of sources discarded after visual inspection. Left: saturated by a close star. Middle: galaxies at the limit of our detection threshold. Right: HII region of a foreground spiral galaxy.

this sample, a total of 69 objects were rejected after a careful visual inspection. Figure 3 shows some examples of fake detections, galaxies saturated by a close star, galaxies at the limit of our detection threshold, and HII regions of foreground spiral galaxies that were removed from the final sample. Our final catalogue contains 220 starburst galaxies. In Fig. 4 (left), we show the distribution in redshift for our sample, including the gaps in redshift for the photometric sample. In Fig. 4 (right) the percentage of photometric and spectroscopic sample, with respect to the total number of objects in COSMOS, is shown. As can be seen, the distribution in redshift in our sample is not homogeneous (as a consequence, our sample distribution does not have a perfect completeness).

\section{K-correction and stellar mass determination}

In this section, we compute the stellar mass for the 220 starburst galaxies of the sample. To account for redshift effects in the flux measurements for every filter of the photometric catalogue, we first calculated the $K$-correction for each galaxy. We used the last
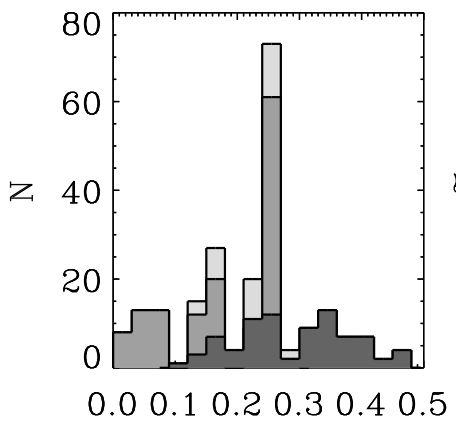

Redshift

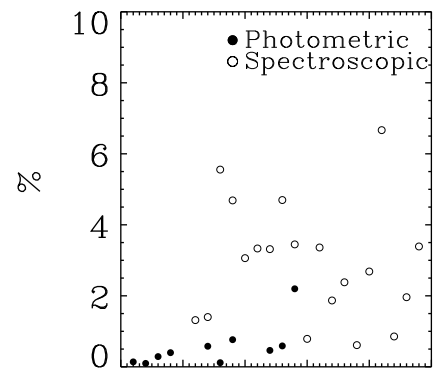

0.00 .10 .20 .30 .40 .5

Redshift
Fig. 4. Left: redshift distribution of the photometric (gray), spectroscopic (dark gray) and total (light gray) sample. Right: percentage of starburst galaxies found in COSMOS, with respect to the total analyzed galaxies in different redshifts. The photometric sample is shown with filled circles, and the spectroscopic sample with open circles.

version of $K$-correct ${ }^{1}$ (Blanton \& Roweis 2007). This software uses a set of 485 spectral templates, in which 450 of these are a set of instantaneous bursts from Bruzual \& Charlot (2003) models, using the Chabrier (2003) stellar initial mass function (IMF) and the Padova 1994 isochrones (Alongi et al. 1991). The remaining 35 templates are from MAPPINGS-III (Kewley et al. 2001), which are models of emission from ionized gas, a crucial feature that appears in the galaxies in our sample. All our galaxies are in the photometric redshift catalogue, and have been mapped by COSMOS using 10 bands $(U, B, V, g, R, I, F 814 W$, $z, J$, and $K$ ). In some cases, the magnitude in one of the bands was not available ( $\sim 10 \%$; the $K$-band usually). In these cases, the $K$-correction was calculated using only nine bands. In our catalogue (Table 3), every magnitude and color are in rest-frame. We visually analyzed every spectral energy distribution (SED) of

1 http://howdy.physics.nyu.edu/index.php/Kcorrect 

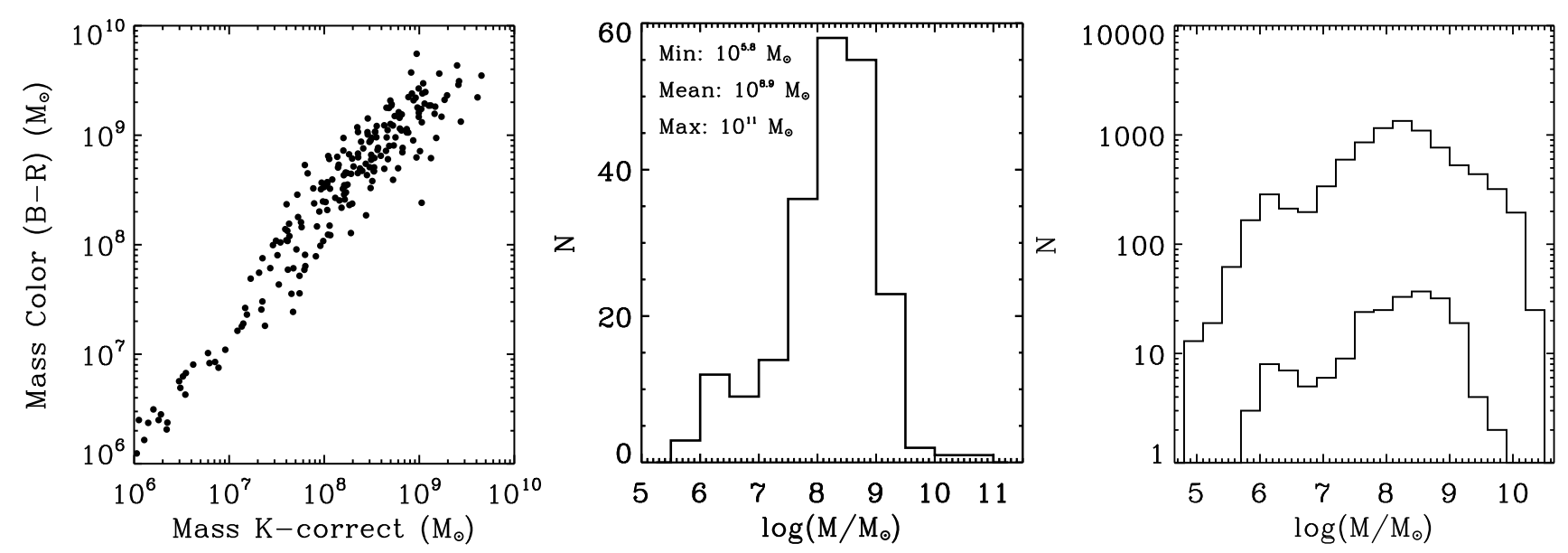

Fig. 5. Left panel: comparison of stellar masses estimated from the $K$-correct software fitting the galaxy SED, and the $B-R$ color using the prescription by Bell \& de Jong (2001). Middle panel: stellar mass distribution of the starburst galaxy sample, determined with $K$-correct. Right panel: stellar mass distribution for both the entire COSMOS galaxy sample with $z<0.3$ and $m(F 814 W)<23.5$ and the starburst identified in our paper (in logarithmic scale).

the sample to check that they are blue emission-line galaxies. To estimate the goodness of the fit, we defined the "goodness parameter" $(\mathrm{G})$, which is expressed as $\sum\left(F_{t}-F_{m}\right)^{2} / F_{t}^{2}$, where $F_{t}$ is the flux of the template at the wavelength of the filter, and $F_{m}$ is the measured flux in the filter from COSMOS. The G parameter is also given in Table 3. The best fits from $K$-correct show some features, such as blue galaxies Balmer break in the continuum and strong emission lines. Two (among the 220) galaxies do not have these features, and the fit is very poor ( $G$ very high). They appear with a value of 99 in Table 3.

The $K$-correct software also provides information about the stellar masses of galaxies. These are published in Table 3, and are in the range $10^{5.8}<M / M_{\odot}<10^{11}$. We note that our selection criteria does not introduce any bias on the mass distribution. The low-mass end of our mass distribution is given by the observational limits $(m F 814 W<23.5)$ whereas the limited volume probed by the COSMOS survey produces the high-mass end. For the sake of comparison, we also computed stellar masses using the equations provided by Bell \& de Jong (2001). They use a suite of simplified spectrophotometric spiral galaxies with SF burst to calculate the stellar mass to luminosity ratio using colors and assuming a universal scaled Salpeter IMF. To apply these equations, we used three independent colors, with magnitudes previously $K$-corrected, to calculate the stellar mass and to check the robustness of our results. The results are consistent with the mass obtained with $K$-correct. Figure 5 (left panel) shows the comparison between the stellar mass from $K$-correct and Bell \& de Jong (2001); both methods to calculate the galaxy mass are consistent. Figure 5 (middle panel) shows the mass distribution of the starburst galaxies determined with $K$-correct. Most of them are in the $10^{8}-10^{9} M_{\odot}$ range. For comparison, we also computed the stellar masses of the whole COSMOS sample with $z<0.3$ and $m(F 814 W)<23.5$ (8650 galaxies) using the $K$-correct algorithm. The result is shown in Fig. 5 (right panel) where both distributions are plotted. It is clear that the number of galaxies in the COSMOS volume at this redshift range peaks at $M / M_{\odot} \sim 2 \times 10^{8}$ with only a few galaxies with $M / M_{\odot}>10^{10}$. The shape of the mass distribution is actually very similar to that of starburst galaxies, with maybe an excess of galaxies in the high-mass end. We suggest that this excess could be related to more red, elliptical-like galaxies dominating this region of the mass function.

\section{Morphology of the starburst galaxies}

The COSMOS field has been targeted by the HST/ACS camera with the $F 814 W$ filter (Capak et al. 2007). This filter is centered at $\lambda_{\mathrm{c}}=8037 \AA$ and its wavelength width is $\Delta \lambda=1862 \AA$. The $F 814 W$ HST/ACS high-resolution images of the sample (220 galaxies), with a Full Width at Half Maximum (FWHM) of the point spread function (PSF) of $0.09^{\prime \prime}$ and a pixel scale of $0.03^{\prime \prime} /$ pixel, are available in the IRSA's General Catalogue Search Service ${ }^{2}$, and have been analyzed to identify morphological structures. A semi-automatic protocol was designed with this aim in mind. We downloaded the images with a size of $15^{\prime \prime} \times 15^{\prime \prime}$ centered on our targets coordinates. Most galaxies are smaller than this size (see Fig. 6 for an example). However, we found that 26 are larger than the downloaded images, and they were analyzed individually. We use Source-Extractor ${ }^{3}$ (Bertin \& Arnouts 1996) to obtain the coordinates of the galaxies and put them in the center of the images. To perform a detailed analysis, particularly to identify sub-structures like star-forming regions in starburst galaxies, we used the Faint Object Classification and Analysis System $\left(\mathrm{FOCAS}^{4}\right)$. This program offers a splitting routine that deals with galaxy compounds well.

With Source Extractor (SExtractor), we define the extension area of the galaxy. For this, through an iterative process, the background on the images was determined to identify objects with a signal higher or equal to three times this value. The minimal area to be considered was imposed to be $3 \times F W H M$ of the HST/ACS images to avoid the detection of spurious sources, such as hot pixels and cosmic rays.

At this stage, different parameters are calculated for the detected objects. In particular, we measured the central position and the equivalent radius, i.e., the radius of a circle with the same area to that covered for the pixels associated with the object. Then the galaxy is relocated in the center of the image using the new position, and the image is resized to eight times its equivalent radius. The outermost isophote was used to obtain the flux, ellipticity, and radius of galaxies which are provided in Table 3.

\footnotetext{
2 http://irsa.ipac.caltech.edu/data/COSMOS/index_ cutouts . html

3 http://www. astromatic.net/software/sextractor 4 http://iraf.noao.edu/ftp/docs//focas/focas.Z
} 

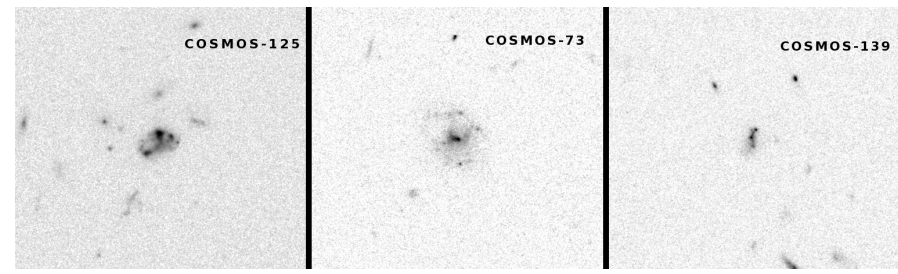

Fig. 6. F814W HST/ACS $15^{\prime \prime} \times 15^{\prime \prime}$ high resolution images of three identified starburst galaxies.
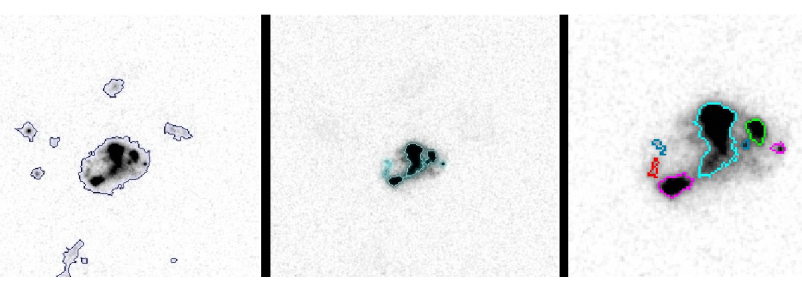

Fig. 7. $F 814 W$ HST/ACS images. Example of the procedure to identify SF knots. Left: first isophotal analysis, where the principal objects in the image are defined. Middle: second isophotal analysis of the target galaxy, where substructures are found. Right: a zoom of the image in the middle. Regions with a radius bigger or equal to the PSF $\left(0.09^{\prime \prime}\right)$ will be identified as subregions or knots. For clarity the detected regions are shown with different colors.

\subsection{Surface brightness analysis and photometry}

With the resized and centered images, we performed an isophotal analysis with FOCAS. First, we calculated the background $\sigma$. Then, the objects are identified where the count level is above three times this value. We used the new position calculated for the objects to identify our target and separate it from other spurious sources that may be present in the field. For this, we define an area centered in the object and enclosed by an isophote with signal above $3 \times \sigma$ (see Fig. 7; left panel). As a result, for each cataloged source we obtain coordinates of the central position, total flux, nearby background, surface brightness, equivalent radius, and ellipticity. In Table 3 we show the apparent and absolute magnitudes, luminous radii, colors and their errors, luminosities in $\mathrm{H} \alpha$ and [OIII] and their errors, equivalent widths in $\mathrm{H} \alpha$ and [OIII], surface brightness, ellipticity, and mass for each galaxy.

\subsection{Resolved star-forming regions and diffuse emission}

From a visual inspection of the galaxies, different star-forming knots embedded in the more diffuse and extended emission are clearly visible in the galaxy images. Further analysis was carried out with FOCAS, with the aim of parametrizing such structures. For this, we find the signal over the diffuse emission of the galaxies, looking for individual regions. Besides, some regions may overlap, and then a criterion has to be defined to separate them. The splitting of merged regions is performed using two main parameters: a minimal area according to the FWHM of the PSF and a signal threshold. The signal threshold is set to be $3 \sigma$ of the local background, which is computed iteratively from pixels that are not part of the object (see Valdes 1982, Sect. 4). A loop is entered incrementing the detection threshold level by $0.2 \sigma$ every time. Then, if at some iteration of the loop there is more than one region, the analysis continues separating each one. The loop finishes when the peak of every region is reached.

In Fig. 7 the process to identify substructures is shown. All starburst galaxies in our sample were morphologically classified

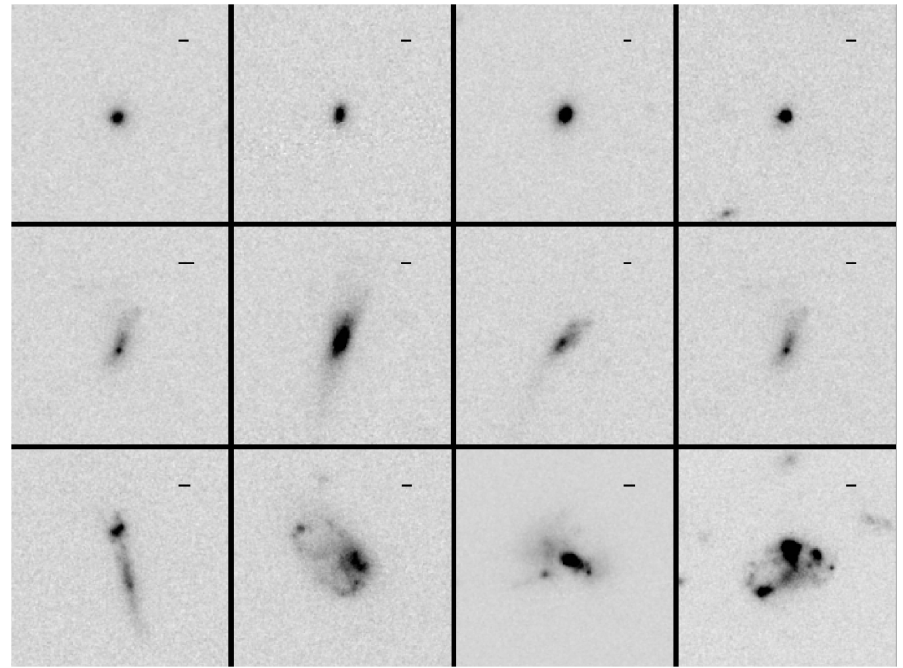

Fig. 8. Example of the starburst morphological classes in our sample, a $1 \mathrm{kpc}$ bar is shown at the top right of each image. Sknot galaxies (top row), Sknot+diffuse galaxies (middle row), and Mknots galaxies (bottom row).

based on this isophotal analysis as follows: Sknot, when it consists of a single knot of star formation; Mknots, when several knots of star formation are identified within the galaxy size; Sknot+diffuse, if the single knot is surrounded by diffuse emission. Figure 8 shows examples of the three morphological classes.

The $F 814 W$ filter was used for the morphological classification. This ensures the $\mathrm{H} \alpha$ emission is covered by the filter for all galaxies in our sample with $z>0.1$. At lower redshift, the clumpy morphological features will be detected by the continuum excess associated with the star-forming regions. The redshift distribution of the different starburst classes is presented in Fig. 9. They display very similar trends and they are also in good agreement with the redshift distribution for the whole sample (see Fig. 4). Therefore, we consider that the filter bandpass is not severely limiting our study.

We measured different parameters for each substructure: area, flux, magnitude, luminous radius, and ellipticity. The luminous radius was determined assuming circular symmetry for the isophotes; this is a good approximation for the core of compact star-forming knots. Figure 10 shows the size distribution of galaxies and knots. The typical radius of the galaxies (Fig. 10a) and the knots of Sknots (Fig. 10b), Sknot+diffuse (Fig. 10c) and Mknots (Fig. 10d) are 1-3 kpc, $1-2 \mathrm{kpc}, 0.5-1.5 \mathrm{kpc}$, and 0.5-1.0 kpc, respectively.

In Table 4 we summarize the parameters of the knots in the starburst galaxies. As explained above, they are catalogued as Sknot, Sknot+diffuse and Mknots. When more than one knot in a galaxy was found, they then appear in the table following their $\mathrm{H} \alpha$ luminosity from higher to lower values. The diffuse luminosity of each clump is obtained from the diffuse light of the galaxy, considering the area of the knot. The mass is calculated using $B-R$ color, using the prescriptions of Bell \& de Jong (2001), assuming the same color for the knots and the host galaxy. Distance to the center, radius, and ellipticity were determined with the isophotal analysis.

In Fig. 11 we show the ellipticity of the galaxies for the different morphologies: Sknot, Mknots, and Sknot+diffuse. It is worth noting that Sknot galaxies are preferentially round, with a mean ellipticity of around 0.4. Galaxies with a single knot and 


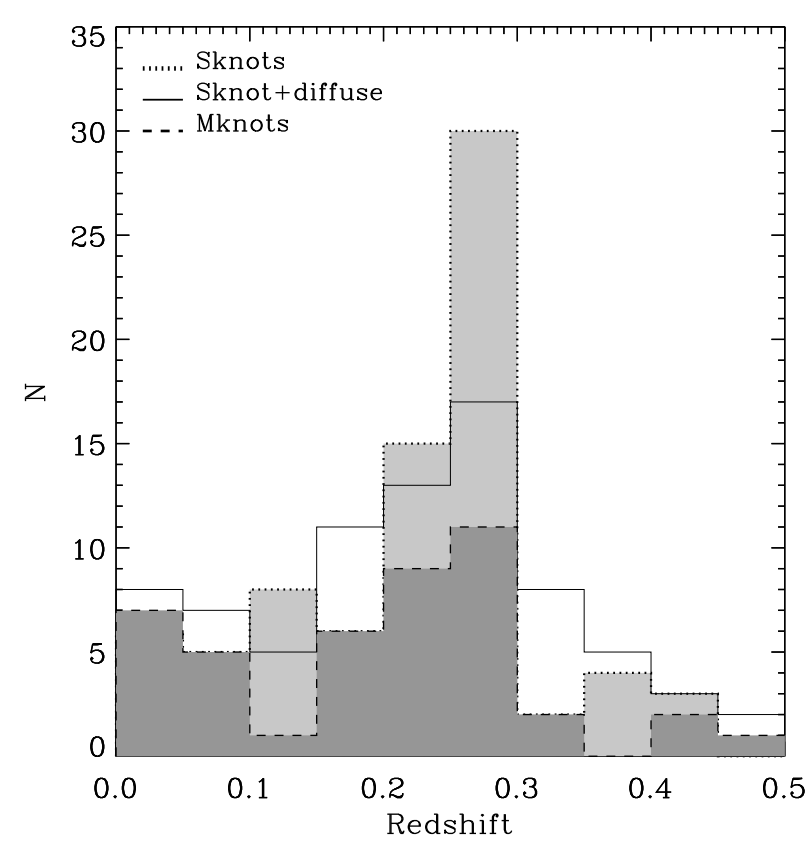

Fig. 9. Redshift distribution for the three starburst morphological classes defined in this paper: Sknot galaxies (light gray), Sknot+diffuse galaxies (empty histogram), and Mknots galaxies (dark gray).
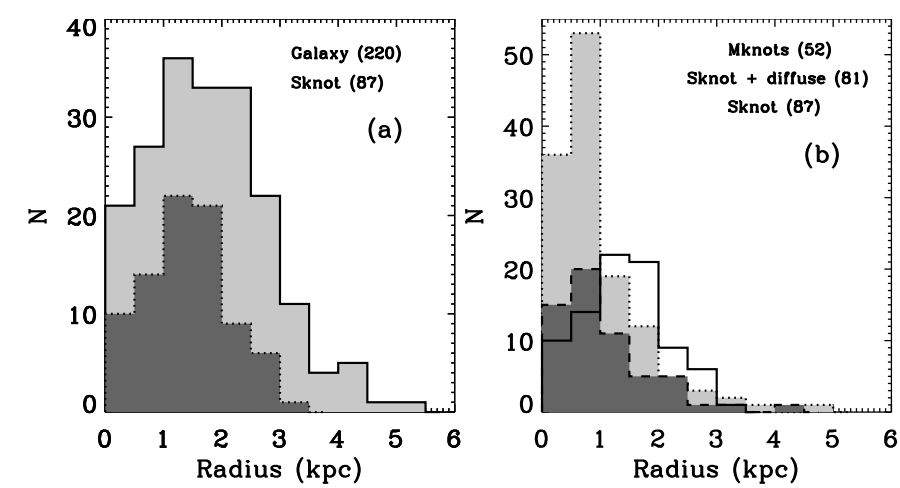

Fig. 10. Size distribution of the galaxies and star-forming knots from the isophotal analysis of the HST high spatial resolution $F 814 \mathrm{~W}$ images. Circular symmetry is assumed to estimate the radius. Panel a), the distribution of both the total sample and Sknot galaxies is shown in light gray and gray, respectively. Panel b), the distribution of knots in Mknot galaxies, Sknot+diffuse galaxies, and Sknot galaxies is shown in light gray, gray, and empty histogram.

diffuse light, and those with multiple knots, are more elongated, with mean ellipticities of 0.63 and 0.65 , respectively.

\subsection{Spatial distribution of the star-forming knots in the galaxies}

The galaxy centers were calculated with SExtractor, using the barycenter of the emission within the outer isophote; the center of the knots correspond to the maximum luminosity of the isophote, and their radii are equivalent to a circular shape of the isophotal area. All the projected linear scales have a resolution of $0.09^{\prime \prime}$ (limited by the PSF). Figure 12 shows the distance to the center of the galaxy for each knot versus its radius. A bisector separates the knots in two classes: knots in the upper region (open circles) are off-centered, whereas in the lower region (filled circles) the knot overlaps with the geometrical center of the galaxy. We call them offcenter and lopsided, respectively. It

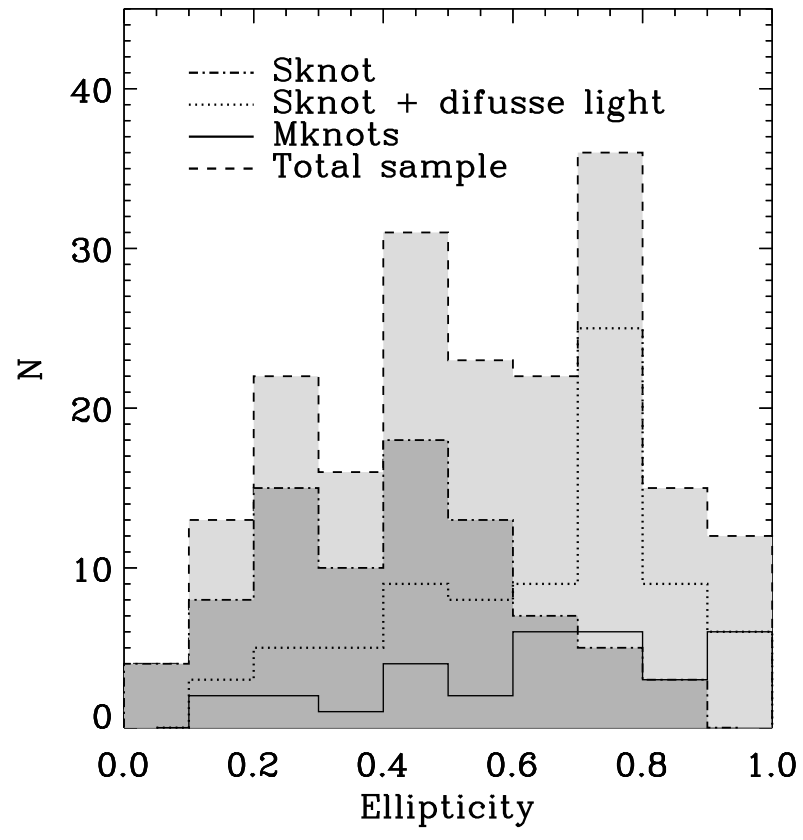

Fig. 11. Distribution of the ellipticity of the galaxies. Four cases are considered: galaxies with Sknot, Sknot+diffuse, Mknots, and the total sample.

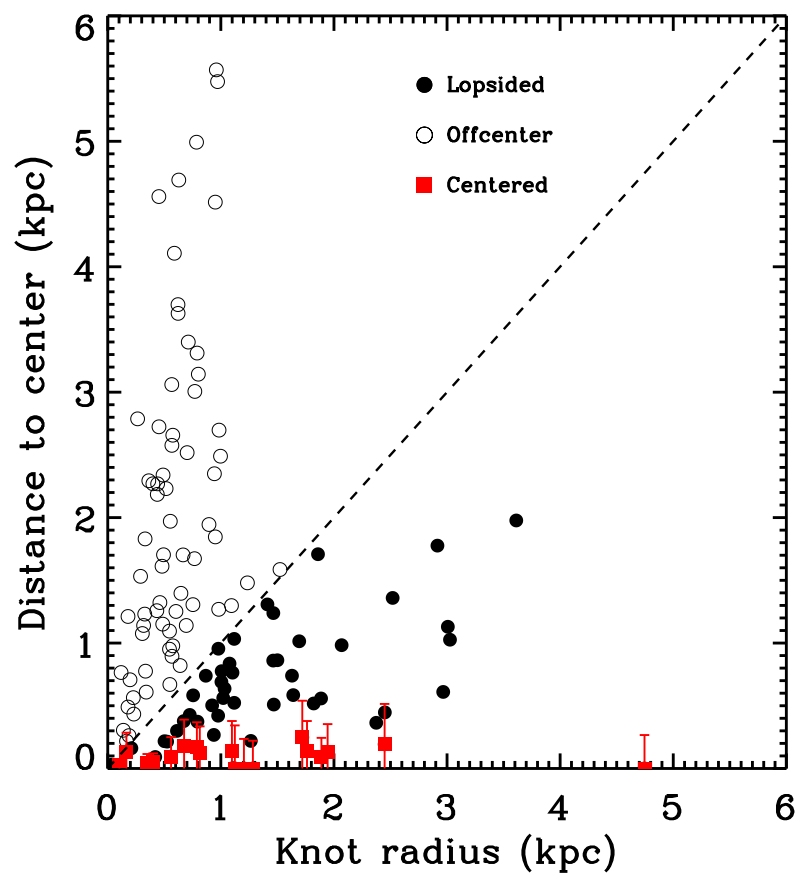

Fig. 12. Knot sizes versus their distance to the center of the galaxy. From the diagram we separate three classes: offcenter (open circles), lopsided (filled circles) and centered (red squares with error bars) regions. A bisector separates two regions. Filled circles show regions in contact with the center of the galaxy and open circles show regions off-centered. Regions which overlap with the geometrical center of the galaxy are labeled "centered" and represented with squared symbols. Overplot bars account for the spatial resolution.

is worth noting that those knots whose distance to the center is lower than their size, taking into account the spatial resolution of the HST, have been labeled as centered and plotted with a square symbol. 


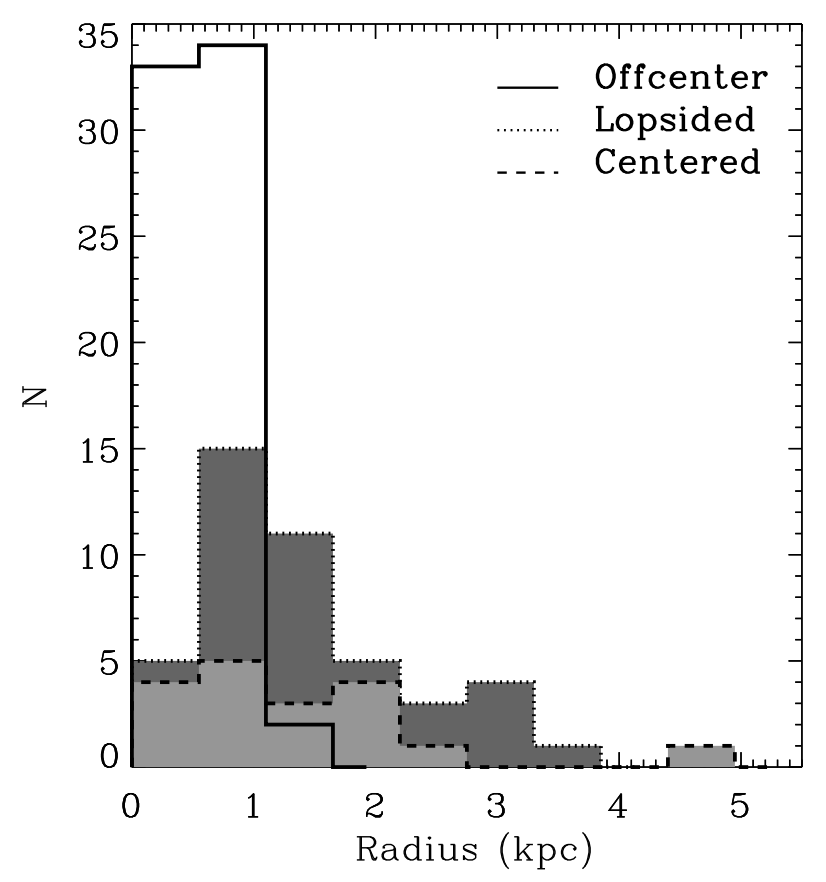

Fig. 13. Size distribution of knots, as identified in Sect. 4.3. Solid (empty), dotted (dark gray) and dashed (light gray) lines show the size distribution of offcenter, lopsided, and centered knots. The largest starforming knots are located in the central regions of the galaxy.

Figure 13 shows the size distribution of the knots. Solid, dotted, and dashed lines show the distribution of offcenter, lopsided, and centered knots. Offcenter knots are smaller and more abundant. Lopsided and centered knots are generally larger but they also span a wider range in sizes. The mean radii are $0.1,0.5$, and $2.1 \mathrm{kpc}$ for offcenter, lopsided, and centered knots, and the mean distances to the center are $1.3,1.4$, and $0.6 \mathrm{kpc}$, respectively.

\subsection{Mass and SFR estimation of the star-forming knots}

Individual star-forming knots/clumps in our galaxy sample cannot be resolved using the broad-band imaging provided by the SUBARU telescope. Therefore, to estimate their masses using the prescriptions given by Bell \& de Jong (2001, see Sect. 3), we need to rely on the HST imaging. Unfortunately, the COSMOS field is only covered in its entirety by the $F 814 \mathrm{~W}$ filter, so a clump-scale color is not directly accessible from the observations. To overcome this problem, we assumed that the knots and the host galaxy have the same color. Then, we used the $F 814 \mathrm{~W}$ band images to measure the luminosity of the individual knots and the SUBARU color to derive their corresponding masses.

To test the influence of our color hypothesis in the derived knot masses, we cross-matched our sample with CANDELS (Grogin et al. 2011; Koekemoer et al. 2011) and 3D-HST (Brammer et al. 2012) surveys. These new HST surveys only cover a small area of the full COSMOS footprint, but they provide imaging using different filters at cluster-scale resolution. We found four starburst galaxies classified as Sknot+diffuse or Mknots galaxies in this search, accounting for a total of 12 knots/clumps of star formation. Using the $F 606 \mathrm{~W}$ filter provided by CANDELS we computed the colors of the individual knots, as well as those of the entire galaxy. The mean value of the rest-frame $F 606 W-F 814 W$ (equivalent to $V-I$ ) color is 0.1 for both the knots and galaxies. However, the knots present a broader range of values $(\sigma \sim 0.5 \mathrm{mag})$. Despite the small sample

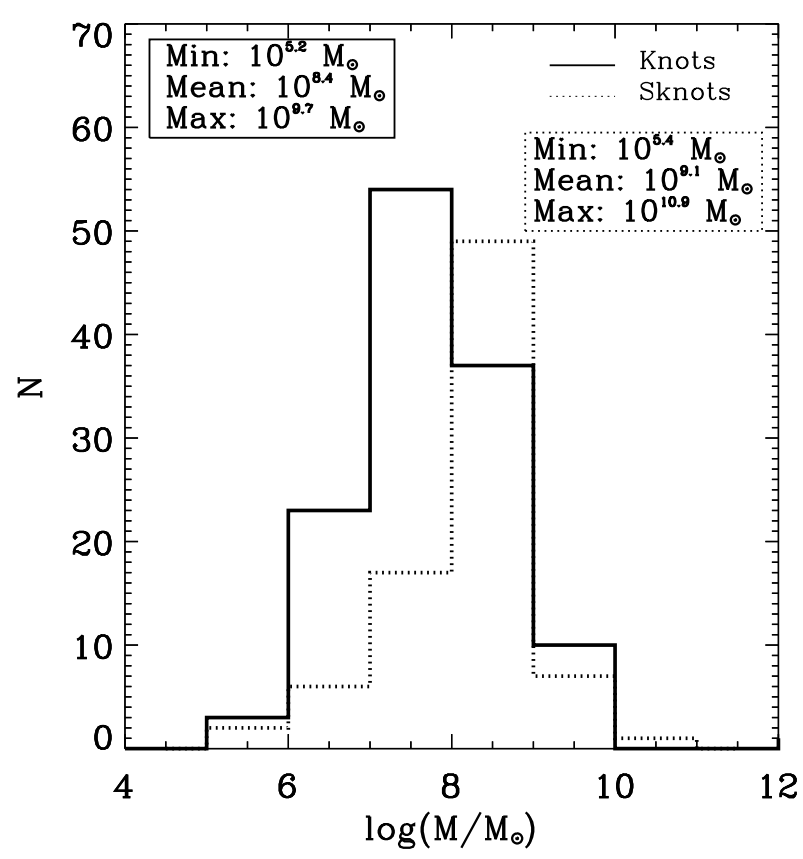

Fig. 14. Mass distribution of the knots in Sknot+diffuse, and Mknots galaxies (solid line) and Sknot galaxies (dashed line). The statistics are given in the boxes with the same line code.

used in this study (only four galaxies and 12 knots), our results are backed up by previous findings in the literature. Wuyts et al. (2012), using a sample of 323 starburst galaxies, claims that no difference in color is found between the disk and clump regions when the clumps are detected using the surface stellar mass as a detection method (see their Fig. 6). Guo et al. (2012) also find a similar result, using a sample of 40 clumps detected using $z$-band images. They claimed that the mean $U-V$ color of clumps is similar to that of disks, with the color distribution of clumps being broader (see their Fig. 5). Therefore, even if for a given knot, the color difference can be up to $0.5 \mathrm{mag}(1 \sigma)$, on average the assumption that the colors of knots and the host galaxies are the same is still valid. To account for the individual variations, we propagated a $1 \sigma$ error in the clump colors to the derived stellar masses. Even so, possible radial color gradients can be present within individual galaxies. Guo et al. (2012) estimated a $U-V$ color difference between center and off-center star-forming knots of $\sim 0.5 \mathrm{mag}$, similar to the $1 \sigma$ scatter for our mean colors. Whether this color gradient is present in our sample or not, it systematically overestimates the stellar mass for bluer knots (bluer than the integrated galaxy) and underestimates it for redder knots by a factor comparable to our $1 \sigma$ error.

Another possible caveat on our knot-mass determination might be the use of different band-passes affecting our results. To estimate how accurate this approximation is, we use our sample of Sknot galaxies. These starburst galaxies do not contain diffuse light and, therefore, the measurement using the higher resolution HST images should agree with those from the ground-based SUBARU photometry. We calculated the mass of the galaxies using the $M / L$ ratio (Bell \& de Jong 2001) with the $B-R$ color (the less affected by the background galaxy), and the luminosity in the SUBARU I-band and HST/ACS F814W-band with a fixed aperture of $3^{\prime \prime}$ (COSMOS catalogue). We find an excellent agreement between both measurements.

The masses of the knots and their corresponding errors are provided in Table 4 and represented in Fig. 14. The mean mass for knots in Sknot+diffuse and Mknots galaxies is $10^{8.4} M_{\odot}$. 


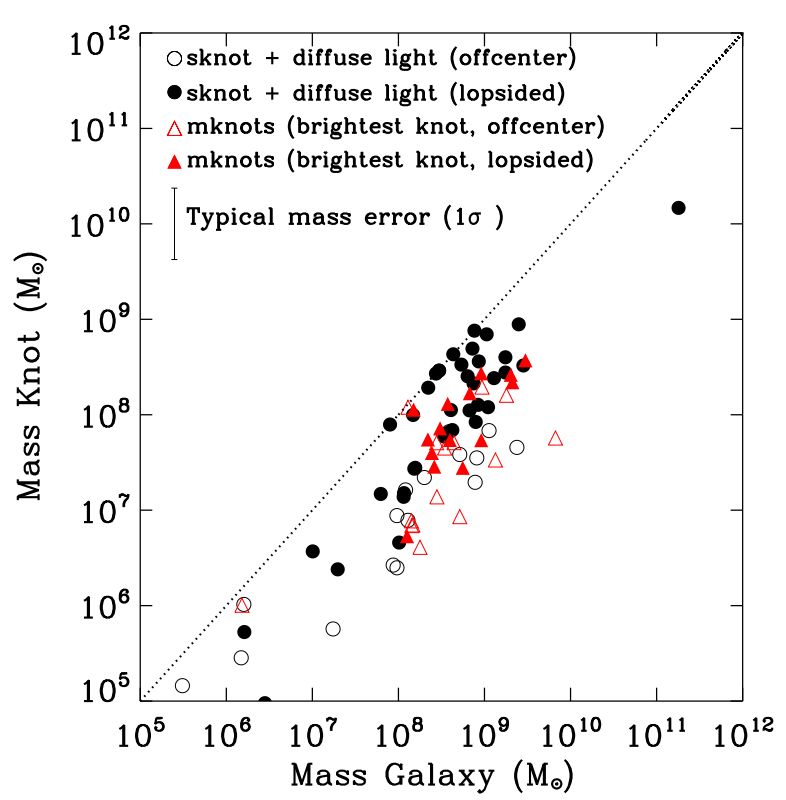

Fig. 15. Comparison of masses of the knots with that of their host galaxies. Symbols represent the different morphologies (code inserted): one knot plus diffuse light, and the brightest knot in multiple knot galaxies. Sknot galaxies are not represented, since they would follow the bisector.

The minimum and maximum are $10^{5.1}$ and $10^{9.7} M_{\odot}$, respectively. We also show the mass distribution of the single knot galaxies (dashed line). We compute a mean mass for Sknots of $10^{9.1} M_{\odot}, 5$ times larger than the mean of knots in Sknot+diffuse and Mknots galaxies. For Sknot+diffuse and Mknots, we compare the masses and sizes of the knots and the galaxies. The mass comparison is shown in Fig. 15. The masses of the knots in Sknot+diffuse and Mknots galaxies correlates with the total galaxy mass, with more massive knots being in more massive galaxies. As previously pointed out, a systematic color gradient in our galaxy can produce differences in the mass estimation of the knots in Mknot galaxies of the order of the typical mass error shown in Fig. 15.

The $\mathrm{H} \alpha$ luminosity of the different star-forming galaxies in our sample was estimated following different strategies. Sknot do not require the HST/ACS spatial resolution to be measured. Therefore, we used the SUBARU intermediate filters to determine the luminosity in $\mathrm{H} \alpha$ and continuum regions. Using these filters, we were able to trace the $\mathrm{H} \alpha$ emission in the redshift ranges $0.01 \leq z \leq 0.2$ and $0.23 \leq z \leq 0.28$. Twenty Sknot galaxies in our sample are in this redshift range, and nine of them have spectra in $z$ COSMOS. We used the difference between photometric and spectroscopic $\mathrm{H} \alpha$ luminosity of these galaxies to estimate our uncertainty, the mean of this difference is $0.66 \mathrm{dex}$.

For Sknot+diffuse and Mknots with $z>0.1$ the $F 814 \mathrm{~W}$ filter includes the $\mathrm{H} \alpha$ emission, the continuum associated with the star-forming region, and the continuum $+\mathrm{H} \alpha$ emission of the diffuse light of the host galaxy. The continuum and $\mathrm{H} \alpha$ diffuse emission from the host galaxy was removed computing the luminosity in an area equivalent to the knot, but in the diffuse gas region of the galaxy and subtracting it from that of the corresponding knot.

The star-forming continuum was subtracted using a statistical value derived from the $\mathrm{H} \alpha /$ continuum ratio for 51 Mknots galaxies with spectrum in $z$ COSMOS. Figure 16 shows the distribution of this so-called correction ratio. The Gaussian fit provides a mean of 0.09 , with a dispersion of 0.05 . We used the mean value to correct the $\mathrm{H} \alpha$ luminosity of the knots and the

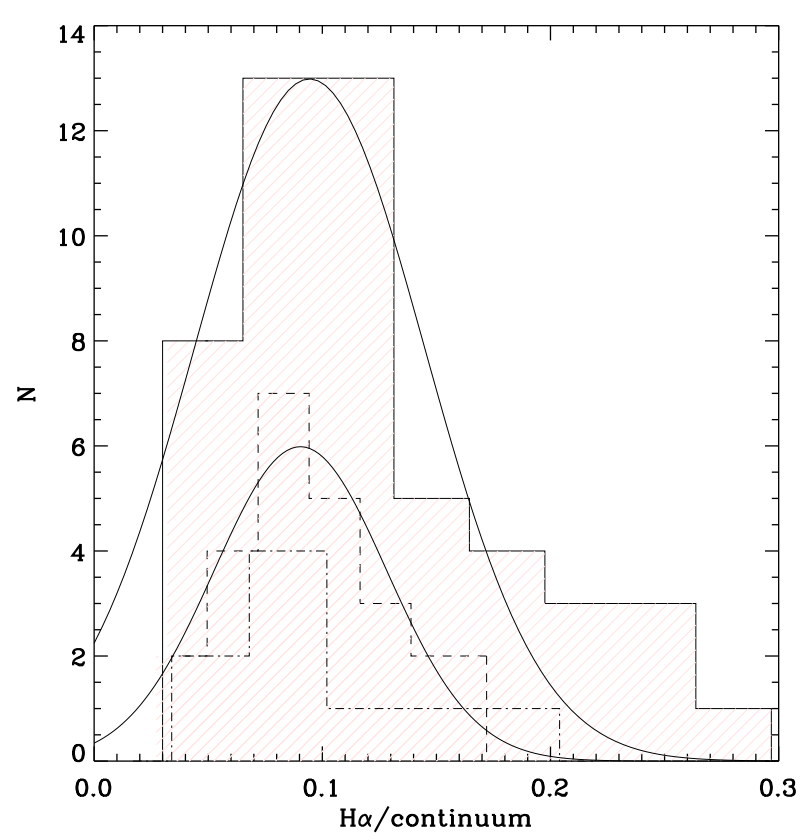

Fig. 16. $\mathrm{H} \alpha /$ continuum distribution for galaxies in our sample with spectra in zCOSMOS. Different lines show: the distribution of the 51 Sknot+diffuse and Mknots galaxies (solid line) with a fitted Gaussian profile overplot; the distribution of a subsample of nine galaxies with bright knots (dot-dashed line), its luminosity being twice or more that of the host galaxy, and the distribution of the 25 Sknot galaxies (dashed line) with the fit of a Gaussian overplot.

dispersion was included in the error budget of the derived quantities (in particular of the SFR). A possible caveat to this method is whether $\mathrm{H} \alpha+$ continuum of the host galaxy could be present in $z$ COSMOS spectra. To check this issue, we used a subsample of galaxies with available spectra and with a star-forming knot/clump luminosity that was at least twice the luminosity of the host galaxy (nine galaxies). For this subsample, we would expect to have a negligible contribution from the host galaxy owing to the different luminosities. We found remarkably similar results using this small sample (see Fig. 16, dot-dashed line). We also analyzed a subsample of 25 Sknot galaxies (galaxies without diffuse light) and with available spectroscopy in zCOSMOS. For this subsample we expected our $\mathrm{H} \alpha$ /continuum distribution to be shifted towards lower values, since there is no contribution from the host galaxy. However, we find again a good agreement with our initial sample of 51 galaxies. Figure 16 (dashed line) shows the derived distribution for Sknot galaxies. The best Gaussian fit to this distribution also provides a mean of 0.09 and a dispersion of 0.04 .

Therefore, we consider that an $\mathrm{H} \alpha$ /continuum correction factor of 0.1 with a typical dispersion of 0.05 is a robust value, even in the most extreme cases in our sample. The final luminosity in $\mathrm{H} \alpha$, obtained as described above for Sknot, Sknot+diffuse and Mknots galaxies, is given in Table 4.

The SFR is calculated from the luminosity in $\mathrm{H} \alpha$, using the relation between $L(\mathrm{H} \alpha)$ and SFR from Kennicutt (1998). Figure 17 shows the radial distribution of the SFR, SFR/area, mass and mass/area for centered, offcenter, and lopsided knots, as defined in Sect. 4.3. As can be seen in Fig. 17 (top), the knots with the highest SFR $\left(\sim 1 M_{\odot} \mathrm{yr}^{-1}\right)$ are only present in the central regions of the galaxies. Figure 17 (bottom) shows the mass and mass/area versus the distance to the center. The more massive and largest star-forming regions are also in the central part of the host galaxies. We note that, if internal color gradients of 

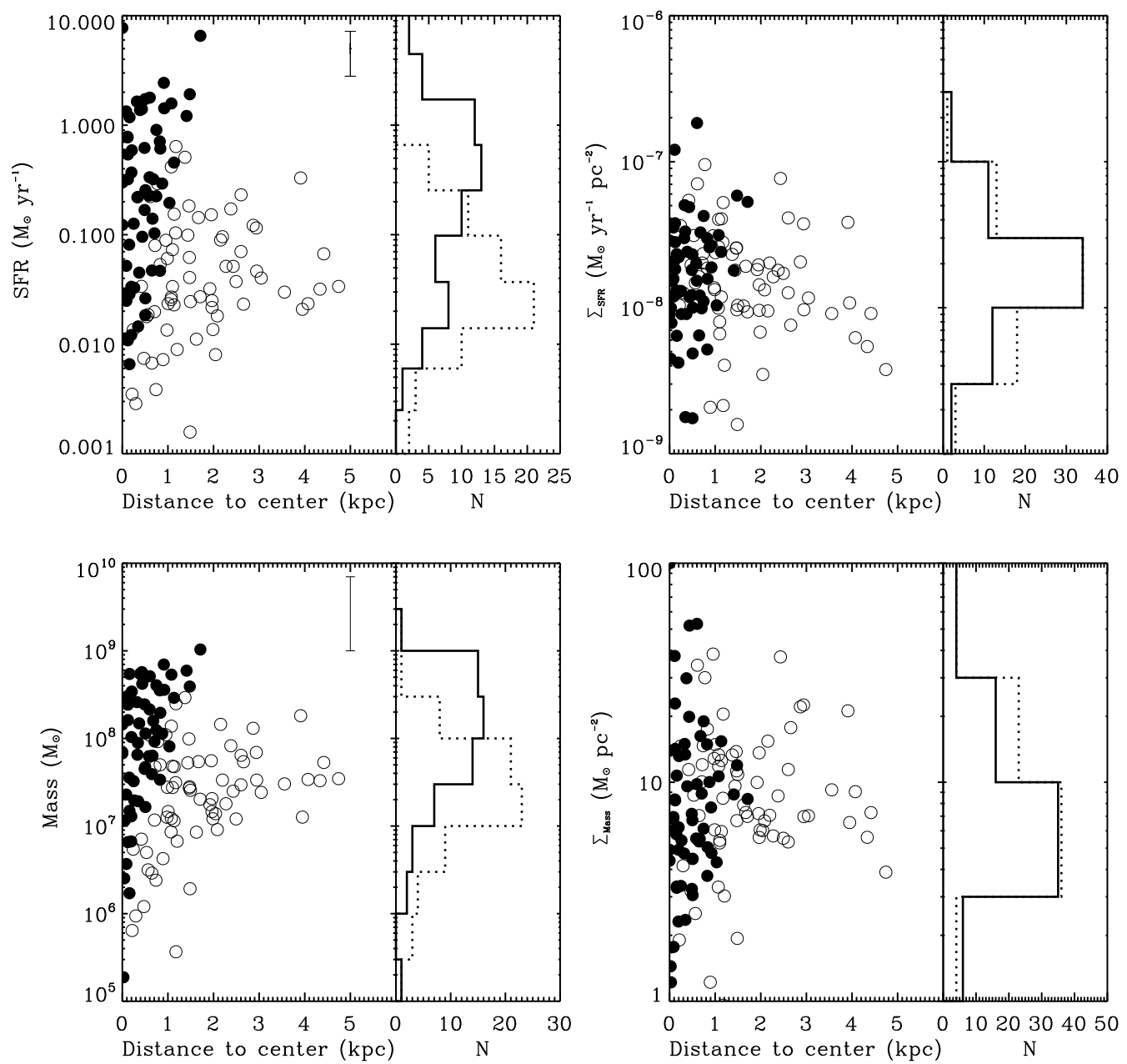

Fig. 17. SFR and $\Sigma_{\text {SFR }}$ versus galactocentric distance (top), and mass and $\Sigma_{\text {mass }}$ versus galactocentric distance (bottom). Filled circles are centered and lopsided knots and open circles represent offcenter knots. The distribution of each quantity in the $y$-axis is shown at the right of each panel, solid line is for centered and lopsided knots, and dotted line is for offcenter knots. As can be seen, the most massive and high star-forming regions are in the central part of the host galaxies. For the sake of clarity, Sknots have not been displayed.

the star-forming knots are present in our sample, the statistical mass difference between both populations (center and offcenter knots) is larger.

\subsection{Single-knot galaxies}

In our catalogue, 87 galaxies were classified as Sknot ( 38\%), meaning that they show a resolved star-forming region and no extended diffuse emission. The minimal area used to define a substructure is 15 connected pixels, with values over $3 \sigma$ the sky background. This gives us a magnitude limit of $\sim 26$. This faint magnitude enables us to detect very low surface-brightness sources. For this reason, and within this magnitude limit, we are confident that Sknot galaxies have no diffuse extended light. Figure 18 shows two examples of the search for substructures in Sknot (left panel) and Sknot+diffuse (right panel) galaxies. The units are in magnitude/arcsec ${ }^{2}$.

Sknot galaxies are larger (see Fig. 10) and more massive (see Fig. 14) than star-forming knots in Sknot+diffuse and Mknots galaxies. However, they have similar $\Sigma_{\mathrm{SFR}}$ and $\Sigma_{\text {mass }}$, implying that they probably share a similar SF mechanism.

The ellipticity distribution of Sknot galaxies shows that they are rounder than other starburst categories, (see Fig. 11). The mean is 0.4 , which is in agreement with spheroidal galaxies

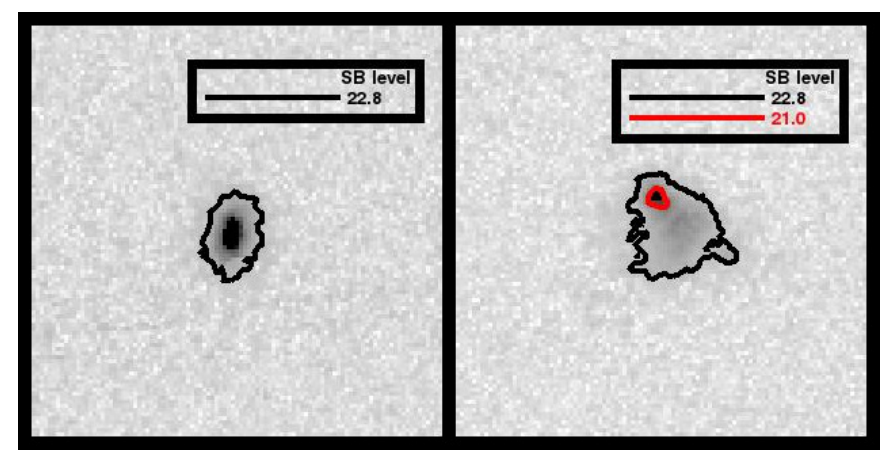

Fig. 18. Substructures in an Sknot (left panel) and Sknot+diffuse (right panel) galaxy. Isophotes are in magnitude/arcsec ${ }^{2}$. The analysis has not found substructures and diffuse light in the Sknot galaxy, while for Sknot+diffuse galaxy it is clear how substructure and diffuse light is found far from the knot region.

viewed from random line of sights (Padilla \& Strauss 2008; Méndez-Abreu 2016).

\subsection{SFR-stellar mass relation}

Figure 19 shows the SFR-stellar mass relation for our sample galaxies, as well as that of individual star-forming knots. SFR 


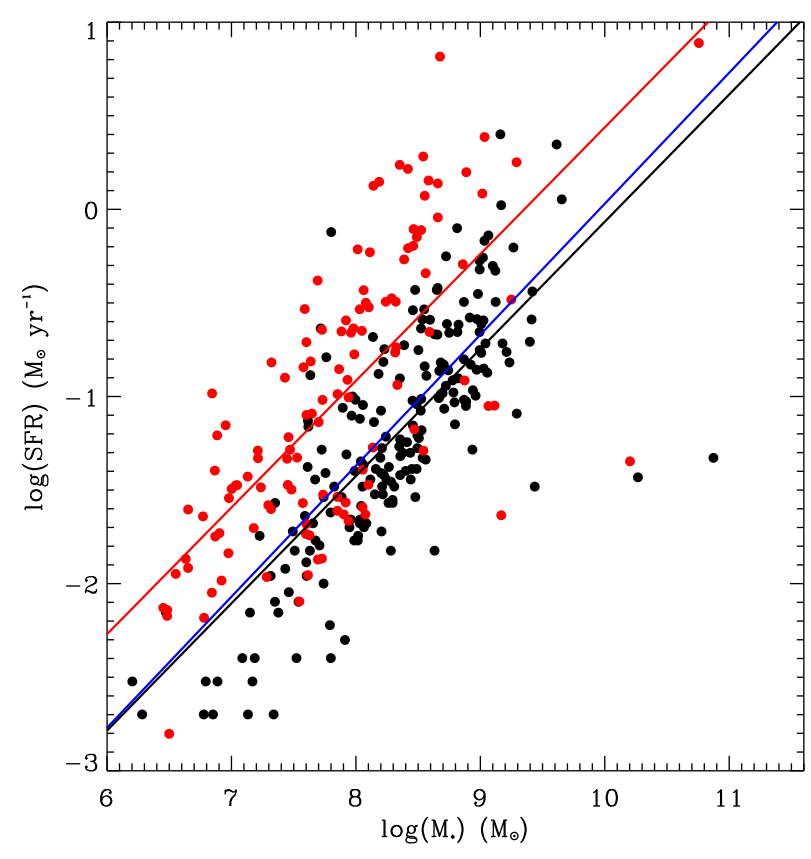

Fig. 19. SFR-stellar mass relation for our sample galaxies (black dots) and star-formation knots (red dots). The SFR-stellar mass relation of each population is fitted by a straight line (black and red). The blue solid line represents the relation of Whitaker et al. (2012a) for star-forming galaxies at $z=0$.

and stellar mass measurements have been described in Sects. 3 and 4 . The best linear fit to the whole galaxy sample and the subsample of star-forming knots is shown in Fig. 19. The slope of the fit for our targets (both the galaxies and the knots) is 0.68 . We compared this relation to that previously presented by (Whitaker et al. 2012a) for star-forming galaxies at $z=0$. The slope of their fit at this redshift is 0.7. We found that our SFRstellar mass relation for the global galaxy properties lies in the locii of other star-forming galaxies studied in the literature. We also found that, even if the clumps follow the same SFR-stellar slope as the galaxies, their SFR is systematically larger by a factor of $\sim 3$. This result is compatible with that presented by (Guo et al. 2012), where they measure the SFR in the knots that resulted in being a factor of 5 higher than that of the underlying disks. We note that the SFR that we measure for the galaxies also includes that corresponding to the starburst knots, which explains why the difference in the SFR (clumps-galaxies) in our data is smaller.

\section{Discussion}

\subsection{Comparison with the literature at this redshift}

The number and properties of star-forming knots in a starburst galaxy provides important information to test the different cosmological models that predict the formation and evolution of galaxies. The availability of recent deep surveys has lead to several works that aim at quantifying the regions in the galaxies where SF is occurring. The precise definition of a starburst knot, however, is very much dependent on the particular observations. A unique definition to search for starbursts in deep fields is still lacking, and the parameters used so far have varied in the different works published in the literature. First studies were done visually (e.g., Cowie et al. 1995; van den Bergh et al. 1996; Abraham et al. 1996; Elmegreen et al. 2004a,b). More recently, Guo et al. (2015) use the excess in UV as the physical parameter to identify a clump. In particular, they classify as a star-forming clump those contributing more than $8 \%$ to the rest-frame UV light of the whole galaxy. Using this definition, they measured the knots in star-forming galaxies in the redshift range $1<z<3$. At lower redshift, $0.2<z<1.0$, Murata et al. (2014) identified the star-forming knots using the HST/ACS F814W-band in the galaxies in the COSMOS survey. The criteria used was that the detected sources must be $2 \sigma$ brighter than the local background, and with a minimum of 15 connected pixels. In this paper, we also used the HST/ACS F814W-band in the COSMOS survey to find and parametrize the star-forming knots of the starbursts identified in the COSMOS survey. In particular, we calculated the emission $(\sigma)$ of the host galaxies and searched for regions with three times this value, which is more strict than the $2 \sigma$ used by Murata et al. (2014), over 15 connected pixels using FOCAS.

The clumpy fraction ( $f_{\text {clumpy }}$; clumpy galaxies/SF galaxies) have been investigated for different redshift and mass ranges (Elmegreen et al. 2007; Overzier et al. 2009; Puech 2010; Guo et al. 2012, 2015; Wuyts et al. 2012; Murata et al. 2014; Tadaki et al. 2014). Despite the already mentioned differences in the clump definition, a remarkable agreement has been reached, concluding that $f_{\text {clumpy }}$ increases with redshift. However, a direct comparison between different works is often difficult, not only because of the clump definition, but also because of the different definition of a starburst galaxy. For example, Guo et al. (2015) and Murata et al. (2014) defined their samples selecting galaxies with specific $S F R(\mathrm{~s} S F R) \geq 0.1 \mathrm{Gyr}^{-1}$. In other works, such as Guo et al. (2012), they used $s S F R \geq 0.01 \mathrm{Gyr}^{-1}$. Wuyts et al. (2012) used galaxies that need less than the Hubble time to form their masses. Tadaki et al. (2014) selected galaxies with $\mathrm{H} \alpha$ excess and Overzier et al. (2009) used Lyman-break ana$\log$ (LBA) galaxies, which share typical characteristics of highredshift Lyman-break galaxies (LBG). On the other hand, Puech (2010) used [OII] emission-line galaxies. The samples are then defined using a variety of criteria. In this paper, we used the values of $E W(\mathrm{H} \alpha)$ and $E W([\mathrm{OIII}]) \geq 80 \AA$ to search for star-forming galaxies. These parameters are used in nearby HII regions and starburst galaxies, and point directly to recent (young) star formation (Kniazev et al. 2004; Cairós et al. 2007, 2009a,b, 2010; Morales-Luis et al. 2011; Amorín et al. 2014).

Using our definition of starburst galaxies, which is based on the EW of the $\mathrm{H} \alpha$ and [OIII] emission lines and the starforming clumps, which is based on light excess in the $F 814 \mathrm{~W}$ filter, we calculated the clumpy fraction for our galaxies. Accounting for our entire sample up to $z \sim 0.5$ we found a value of $f_{\text {clumpy }}=0.24$. The mass range of the galaxies in our sample is $10^{6}-10^{9} M_{\odot}$ (Sect. 3), while in previous studies these values are usually higher (Overzier et al. 2009, $10^{9}-10^{10} M_{\odot}$ ), (Puech 2010, >2 $\times 10^{10} M_{\odot}$ ), (Guo et al. 2012, $>10^{10} M_{\odot}$ ), (Wuyts et al. 2012, $>10^{10} M_{\odot}$ ), (Guo et al. 2015, $10^{9}-10^{11.5} M_{\odot}$ ), (Tadaki et al. 2014, $10^{9}-10^{11.5} M_{\odot}$ ), (Murata et al. 2014, >10 ${ }^{9.5} M_{\odot}$ ). The value obtained in this work for $f_{\text {clumpy }}$ is larger than the value 0.08 found by Murata et al. (2014) at $z=0.3$. Besides the differences in the definition of the knots, the lack of agreement in the value of $f_{\text {clumpy }}$ can be explained by the difference in the mass range of the galaxy sample. On the other hand, Murata et al. (2014) define as clumpy a galaxy with three or more knots, while we considered galaxies with two or more knots. We note that other caveats discussed throughout this paper, such as bandpass or spatial resolution effects, would only increase our clumpy fraction.

Elmegreen et al. (2013) compare the properties of individual clumps of three different galaxy samples: knots in local spiral 

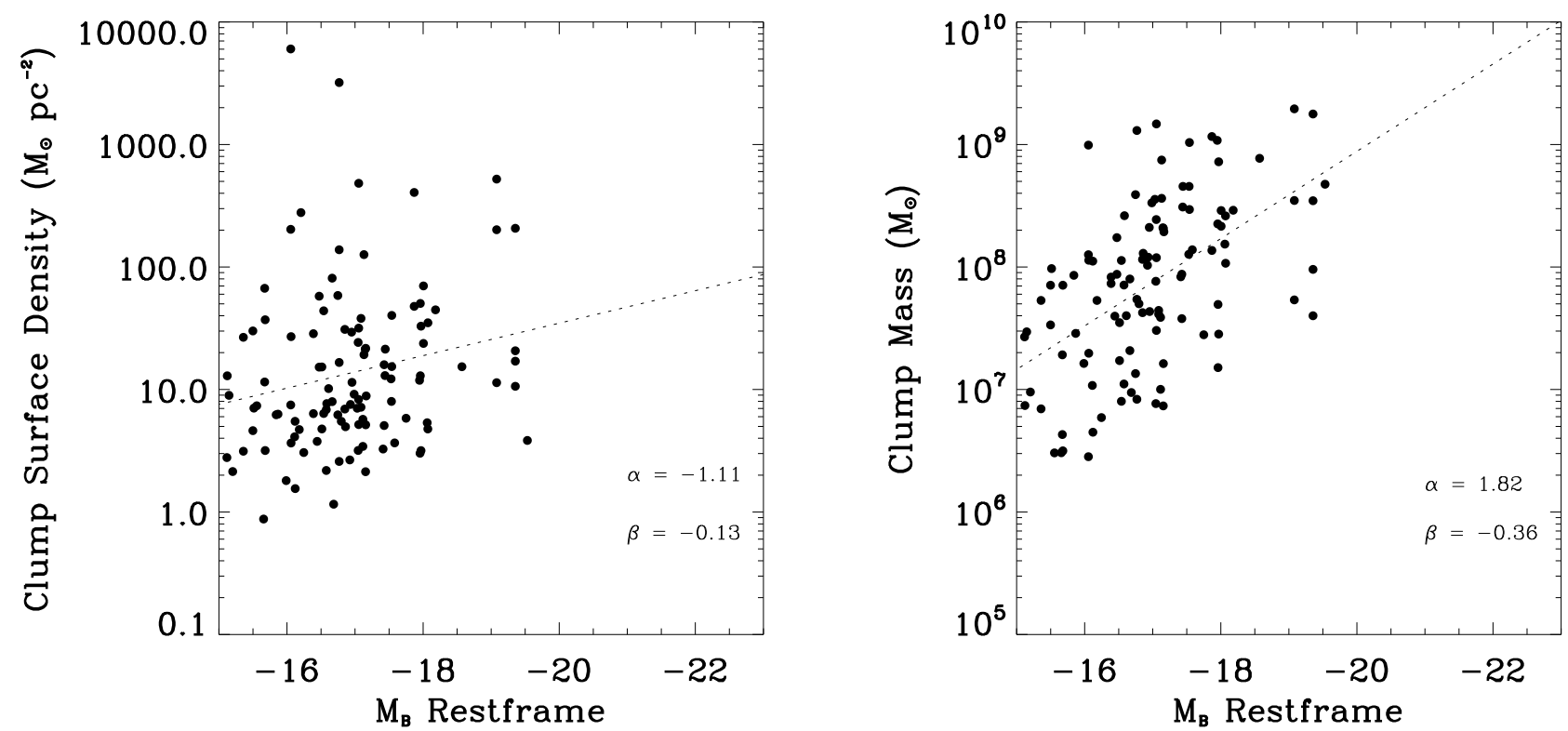

Fig. 20. Clump surface density (left) and clump mass (right) versus absolute $B$-band magnitude of their host galaxy. The best fit to the data is shown in dotted lines. The values of slopes and intercepts are giving in the lower right corner.

galaxies (obtained from the Sloan Digital Sky Survey; SDSS) massive clumps in local starbursts belonging to the Kiso Survey (Miyauchi-Isobe et al. 2010), and clumps in galaxies at high redshift from the Hubble Ultra Deep Field (HUDF). They find correlations between different parameters of the clumps and the absolute $B$-band magnitude of the host galaxy. For a comparison with Elmegreen et al. (2013), we used two properties of our sample knots: surface density and mass. In Fig. 20 we plot them as a function of absolute $B$-band magnitude of the galaxy. These plots are similar to those shown in Figs. 4 and 6 in Elmegreen et al. (2013). The best fit was determined for each parameter, and in the lower right corner of the figures the values of the intercept and slope are given. The surface density and mass trends are in between those provided in Elmegreen et al. (2013) for local and high-redshift massive clumps. The mass versus absolute $B$-band magnitude slopes for Kiso, HUDF, and our sample are -0.54 , -0.18 , and -0.36 , respectively. For surface density versus absolute $B$-band magnitude, the values are $-0.18,-0.12$, and -0.13 . The values suggest that, for a given absolute $B$-band magnitude of the galaxy, the mass and surface density of the knots of the sample of this paper have higher values than those of clumps in local spirals, and lower values than those found in high-redshift, massive star-forming regions. There is a shift in the $y$-intercept of the linear regression fit of our data points, which has higher values than those provided in Elmegreen et al. (2013). This might be due to the different definitions used to determine the area to retrieve the mass, surface density, and SFR.

\subsection{Scaling relations}

Scaling relations are a useful tool for determining parameters when the spectral or spatial resolution is a limitation. A particular case of interest in this work is when the spatial resolution is not good enough to resolve the star-forming regions. In this case, it is usual to rely on the $L(\mathrm{H} \alpha)$ vs. diameter relation to estimate the size of the region we are interested in. Using this reasoning, we assume that the same physical mechanisms and conditions can be applied to star-forming regions throughout a large range of luminosities and sizes.
In our sample, we took advantage of the HST high spatial resolution images, which enable us to spatially resolve the clumps of SF, providing an accurate estimation of their sizes. Furthermore, it enables us to estimate the $L(\mathrm{H} \alpha)$ for individual clumps and explore the $L(\mathrm{H} \alpha)$ vs. diameter relation without further hypotheses. We estimated the $L(\mathrm{H} \alpha)$ for clumps in galaxies with $z \geq 0.1$, as explained in Sect. 4.4. With these values, we plotted the $L(\mathrm{H} \alpha)$ vs. diameter to explore this scaling relation.

Figure 21 shows the $\mathrm{H} \alpha$ luminosity versus diameter of knots in our sample. Blue points are Sknot galaxies. Some Sknot galaxies do show field objects within the fixed SUBARU aperture $\left(\phi=3^{\prime \prime}\right)$. These objects are shown enclosed by a circle and have been discarded for the fit. Sknot with spectra in zCOSMOS were used to estimate errors associated with the luminosities, as was explained in Sect. 4.4. Green points represent these objects, and the error bar is the difference between the spectroscopic and photometric $\mathrm{H} \alpha$ luminosity. The mean value of this difference is taken as the error for the blue points.

The continuum-corrected $\mathrm{H} \alpha$ emission for Sknot+diffuse and Mknots is also represented in Fig. 21 (red points). The errors associated with each knot were computed by propagating the uncertainty in the continuum correction, as explained in Sect. 4.4. To estimate how the uncertainties associated with these $\mathrm{H} \alpha$ measurements can influence the best-fit of the scaling relation, we ran a set of Monte Carlo experiments. We created 100 simulated distributions of $\mathrm{H} \alpha$ luminosity of the knots. Each individual galaxy was allowed to vary its luminosity within the $1 \sigma$ correction obtained from Fig. 16. The best-fit for each distribution was obtained, and it is represented in Fig. 21 (red line). The mean slope and dispersion obtained from this method was 2.46 and 0.04 , respectively.

The value of the slope is an important parameter to understand the universality of the $L(\mathrm{H} \alpha)$ vs. diameter scaling relation. In our sample we found the relation $\log \left(L_{\mathrm{H} \alpha}\right)=(32.2 \pm$ $0.1)+(2.48 \pm 0.05) \cdot \log (d)$ for Sknot, Sknot + diffuse and Mknots galaxies. Fuentes-Masip et al. (2000) obtained a value of 2.5 for the slope for giant HII regions in NGC 4449, and Wisnioski et al. (2012b) found a value of 2.78 using local giant HII regions and high-redshift clumps. 


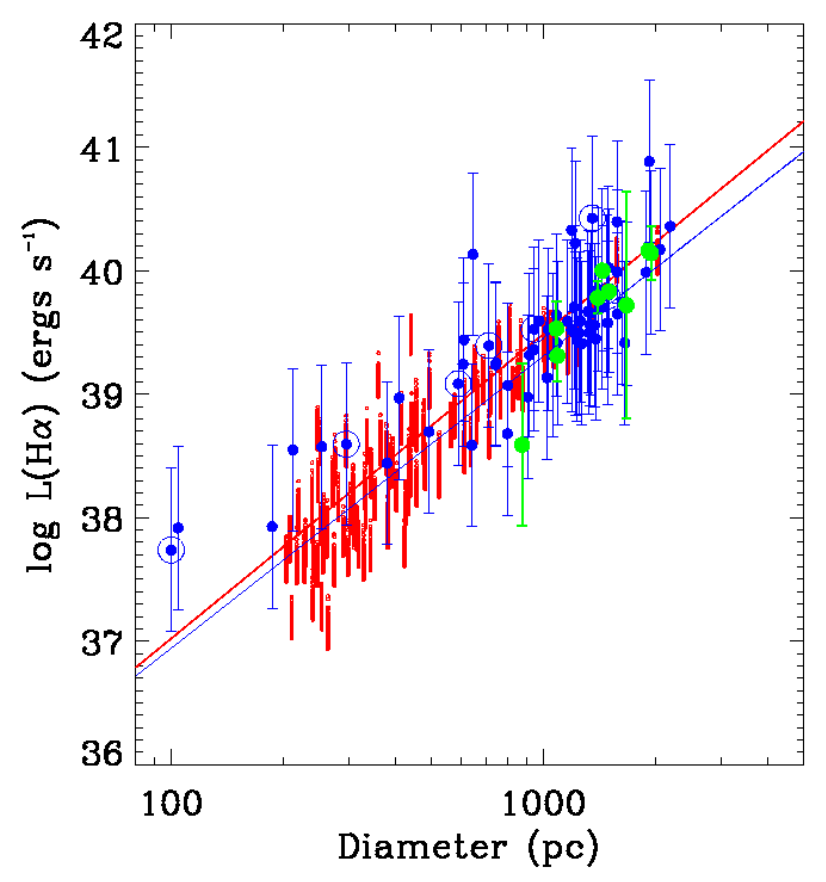

Fig. 21. $L(\mathrm{H} \alpha)$ versus diameter relation. Blue points are Sknot galaxies. Circled enclosed points were discarded for the fit because of contamination in the flux (see Sect. 6.2). Green points are Sknot, with $L(\mathrm{H} \alpha)$ determined photometrically and spectroscopically; the error bar is the difference in luminosities. The mean value of the errors is taken as proxy error for the measurements (error bar for blue points). Red points are estimations of $L(\mathrm{H} \alpha)$ of clumps with a Monte Carlo simulation. The solid red line is the best fit for red points and solid blue line is the best fit for Sknot galaxies.

\subsection{Mknots galaxies and their link to bulge formation}

Several mechanisms have been proposed to explain the formation and evolution of galaxy bulges (Kormendy \& Kennicutt 2004; Bournaud 2016). At high redshift, bulge formation is thought to be driven by either gravitational collapse (Eggen et al. 1962), major mergers (Hopkins et al. 2010), or the coalescence of giant clumps (Bournaud et al. 2007). This latter scenario, in which massive star-forming clumps migrate from the outer disk to the galaxy centre forming a bulge, has been extensively discussed using numerical simulations (Noguchi 1999; Immeli et al. 2004a,b; Bournaud et al. 2007; Elmegreen et al 2008; Ceverino et al. 2010). Recently, Mandelker et al. (2014) used cosmological simulations to provide clump properties to be compared with observations. In particular, they separated clumps formed in situ (clumps formed by disk fragmentation owing to violent instabilities) and ex situ (clumps accreted through minor mergers), finding differences in their properties and radial gradients that could help to distinguish their origins.

In this scenario, Mandelker et al. (2014) predict that in situ clumps show a radial gradient in mass, with more massive clumps populating the central regions. The aforementioned theoretical studies were focused on massive galaxies. More recent analysis deal with less massive galaxies by means of similar theoretical simulations. In Ceverino et al. (2016), they follow gas inflow that feeds galaxies with low metallicity gas from the cosmic web, sustaining star formation across the Hubble time. Interestingly, their results show clump formation in galaxies with stellar masses of $M \simeq 10^{9} M_{\odot}$ using zoom-in adaptive mesh refinement (AMR) cosmological simulations. Altough the cosmic baryonic accretion rate for high-mass galaxies significantly drops from $z \sim 2$ to $z \sim 0$ (see Dekel et al. 2013), the cold flow accretion into low mass galaxies does not dramatically change with redshift. The simulations in Ceverino et al. (2016) with cold gas inflows reaching the galaxy and feeding the SF in galaxies of masses $\sim 10^{9} M_{\odot}$ are the only available simulations so far. They are a good example to compare with observational information that is provided in this paper.

Figure 17 shows the distribution of mass and mass surface density for our clumps as a function of the galactocentric distance. Despite the errors introduced by the lack of multi-band photometry at cluster-scale resolution, it is clear how the most massive clumps in our sample are located in the galaxy center, with offcenter clumps being less massive. However, this tendency is not so clear in terms of surface mass density, where some off-centered clumps have similar surface mass densities to those located in the center, thus likely implying an ex situ formation. On the other hand, numerical simulations also predict that more massive clumps of SF should appear in more massive galaxies (Elmegreen et al. 2008). This is nicely reproduced in Fig. 15, and it is, therefore, consistent with most of the clumps being formed at large radii, and then accreting mass from the disk as they migrate inwards.

Figure 17 also shows the distribution of SFR and SFR surface density versus galactocentric distance. Centered clumps show slightly higher SFR than off-centered ones. This behavior is expected in the case of in situ formation of the clumps (Mandelker et al. 2014). However, the fairly constant radial SFR surface density, also shown in Fig. 17, and predicted for both scenarios, prevented us from extracting further conclusions.

Figure 19 shows the SFR-stellar mass relation for the galaxies and the individual star-forming knots. The figure also includes the best linear fit to both samples and the one given by (Whitaker et al. 2012a) for star-forming galaxies. The SFRstellar mass relation for the galaxies lies in the locii of other star-forming galaxies studied in the literature. The clumps follow the same SFR-stellar trend with the same slope, but shifted systematically by a factor of $\sim 3$. This result is consistent with (Guo et al. 2012) who report an SFR in the knots which is a factor of 5 higher than that of their underlying disks.

In our sample we calculated the sSFR for each knot for every Sknot+diffuse and Mknots galaxy. Figure 22 shows the distribution of sSFR for knots in Sknot+diffuse and Mknots galaxies in our sample, with a bin width of $250 \mathrm{Myr}^{-1}$. We also explored the sSFR for clumps which are centered and off-centered in Sknot+difffuse and Mknots galaxies. The values are similar, and no different SF mechanism can be identified, based on the sSFR. Sknots and knots in Sknot+diffuse and Mknots galaxies have similar values of sSFR; the similarity in the distribution for both samples suggests the same mechanism of SF.

\subsection{Two populations: the knots that are galaxies and the remaining}

In our sample we detected two kinds of star-forming knots: Those that are classified as Sknot galaxies, where there is one star-forming knot, and it is not possible to detect diffuse emission. The others are knots which belong to galaxies with one or more knots of SF and diffuse emission. We detected similitudes and differences in the properties of both classes. Figure 22 represents the distribution of sSFR of both populations, showing a maximum at the same sSFR value. 1/SSFR can also be used as an estimator to determine the time to form the stars that we now see if they were formed at the present SFR. With this idea, the typical time required to form both systems - starbursts spread along 


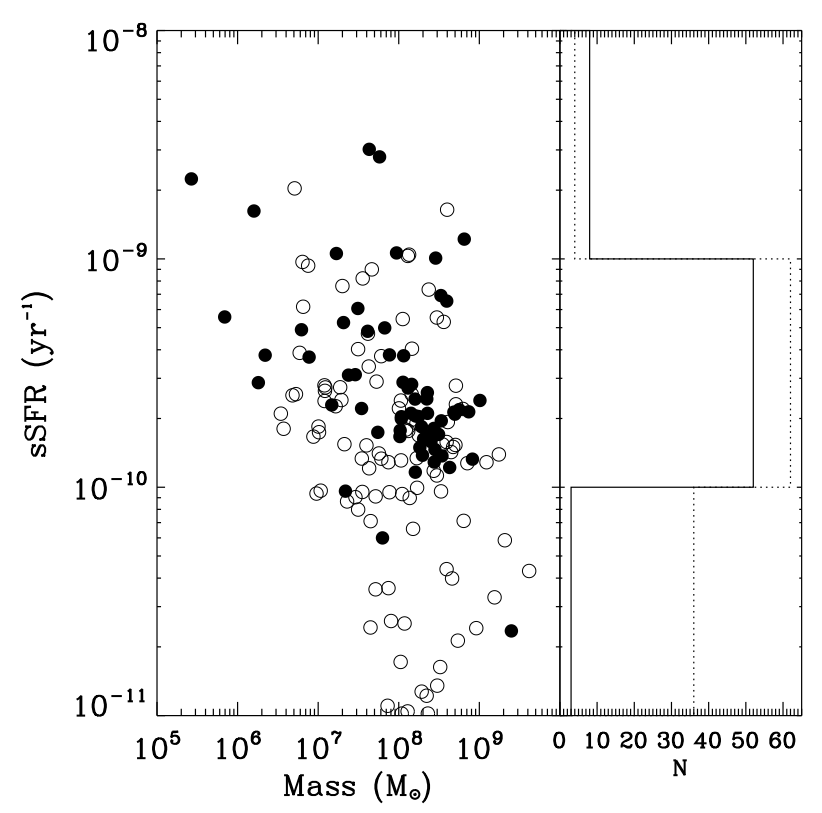

Fig. 22. sSFR versus mass for Sknot (filled circles) and knots in Sknot+diffuse and Mknots galaxies (open circles). To the right, the distribution function (the solid line is for Sknot galaxies and the dotted line is for knots in Sknot+diffuse and Mknots galaxies).

the disks or centered isolated ones - is around $2 \times 10^{9}$ years. Knots in Sknot+diffuse and Mknots galaxies do show, however, a wider range of values, with some cases requiring a formation time of up to $10^{11}$ years. This can be, however, a caveat in our determination of parameters, as explained in Sect. 19.

For both classes, we measured the surface brightness in the $F 814 W$-band. Sknot have lower values, characteristic of low surface-brightness galaxies. For Sknot galaxies, we measured the surface brightness and absolute magnitude in the $B$ - and $V$-band, to compare with the results in the literature. In particular, we compare the photometric values of Sknot galaxies with those provided in Amorín et al. (2012, Fig. 9) and Kormendy \& Bender (2012, Fig. 7), respectively. In Fig. 23a we show the surface brightness in $B$ band versus $B$-band absolute magnitude for Sknot galaxies as in Amorín et al. (2012), and Fig. 23b shows the surface brightness in $V$ band versus $V$-band absolute magnitude as in Kormendy \& Bender (2012). At the right lower corner of each panel, and using the same scale, we show the results provided by Amorín et al. (2012, Fig. 9) and Kormendy \& Bender (2012, Fig. 7). In the figures we overplotted color elliptical regions where most of our points are in the plots of Amorín et al. (2012) and Kormendy \& Bender (2012). A direct comparison suggests that Sknot galaxies fall in the parameter space populated by spheroidals and ellipticals (Kormendy \& Bender 2012), and dwarf elliptical and dwarf irregular galaxies, as measured by Papaderos et al. (2008) and compiled by Amorín et al. (2012).

\section{Conclusions}

We have selected a sample of young starburst galaxies in the COSMOS field using a new tailor-made color-color diagnostic. The final catalogue consists of 220 galaxies with $E W \geq 80 \AA$ in both $\mathrm{H} \alpha$ and [OIII]. The sample was identified by using both the spectra from the $z$ COSMOS catalogue in the redshift range $0.1 \leq z \leq 0.48$, and the photometric redshift catalogue using the
SUBARU intermediate band filters in the redshift ranges $0.007 \leq$ $z \leq 0.074,0.124 \leq z \leq 0.177$ and $0.23 \leq z \leq 0.274$.

To characterize the morphology of the star-forming regions in our sample galaxies, we perform an isophotal analysis of the HST/ACS high spatial resolution images. From this analysis, we classify the starburst galaxies in COSMOS $(z<0.5)$ as Sknot galaxies, Sknot+diffuse galaxies, and Mknots galaxies, whether they consist of a single knot of star formation, a single knot surrounded by diffuse emission, or several knots of star formation, respectively. The stellar masses of the knots and the galaxies were calculated using photometric and spectroscopic data from the COSMOS database. The mean and maximum mass of the starburst galaxies are $10^{8.9} M_{\odot}$ and $10^{11} M_{\odot}$. We have compared this distribution with that obtained for the whole sample of galaxies in COSMOS at the same redshift range. The resulting mean mass is $10^{9}$ with a (similar) lack of galaxies with $M / M_{\odot}>10^{10}$. Therefore, the mass distribution of the starburst galaxies follows the same distribution as the whole sample of galaxies in COSMOS.

The masses for individual knots in Sknot+diffuse and Mknots galaxies vary with the distance to the center of the galaxy, increasing their mass the closer to the center they are. Masses of knots are typically one order of magnitude below that of the host galaxy, peaking at $10^{7.7} M_{\odot}$ (see Fig. 12). The specific characteristics of the starburst knots as a function of their distance to the center of their host galaxy are similar. The surface SFR and surface mass do not show any footprint of their particular location in the galaxy.

The SFR of the knots follows the same trend with their mass to that of their host galaxy, with an offset to higher values of the SFR of the knots of about a factor 3. This tendency is similar to that found by Guo et al. (2012) for higher $z$ galaxies.

Even though the observational criteria to define clumps differs from author to author, and no direct comparison with other published papers can be made, we have tried to define a "clumpy fraction". This parameter, defined in Sect. 5.1, gives a value of $f_{\text {clumpy }}=0.24$, which is higher than other studies (e.g. Murata et al. 2014, $f_{\text {clumpy }}=0.08$ ). The reasons for this difference may be lie in the different mass ranges of the samples, and the precise criteria to identify starburst galaxies and starformation clumps, as we showed in Sect. 5.1.

The fraction of clumpy galaxies is a prediction of the numerical simulations. This is expected to first increase with redshift until $z>4$, where simulations predict less clumpy and more compact star-forming galaxies than at lower redshifts (Ceverino et al. 2015). This is consistent with UV observations of bright clumps in high- $z$ galaxies (Guo et al. 2015), where they found the fraction of clumpy galaxies 0.6 with stellar masses of $\log \left(M / M_{\odot}\right)=9-10$ in the redshift range $z=0.5-3$ (see also Elmegreen et al. 2007 and Tadaki et al. 2014). From this work, the clumpy fraction drops down to 0.24 at $z<0.5$, for the starbursts in COSMOS, which have a typical stellar mass of $10^{8.9} M_{\odot}$. Our result would therefore also agree with the expected trend in the fraction of clumpy galaxies with $z$ predicted by the numerical simulations.

Different properties of the knots, with respect to the absolute $B$-band magnitude of their host galaxies in our sample were compared with local and high-redshift galaxies (Elmegreen et al. 2013). We found that the clump surface density and clump mass versus host galaxy $B$-band absolute magnitude have a slope between those for the local and high-redshift sample, implying that for a given absolute $B$-band magnitude of the galaxy, the mass and surface density of the knots of the sample of this paper have 

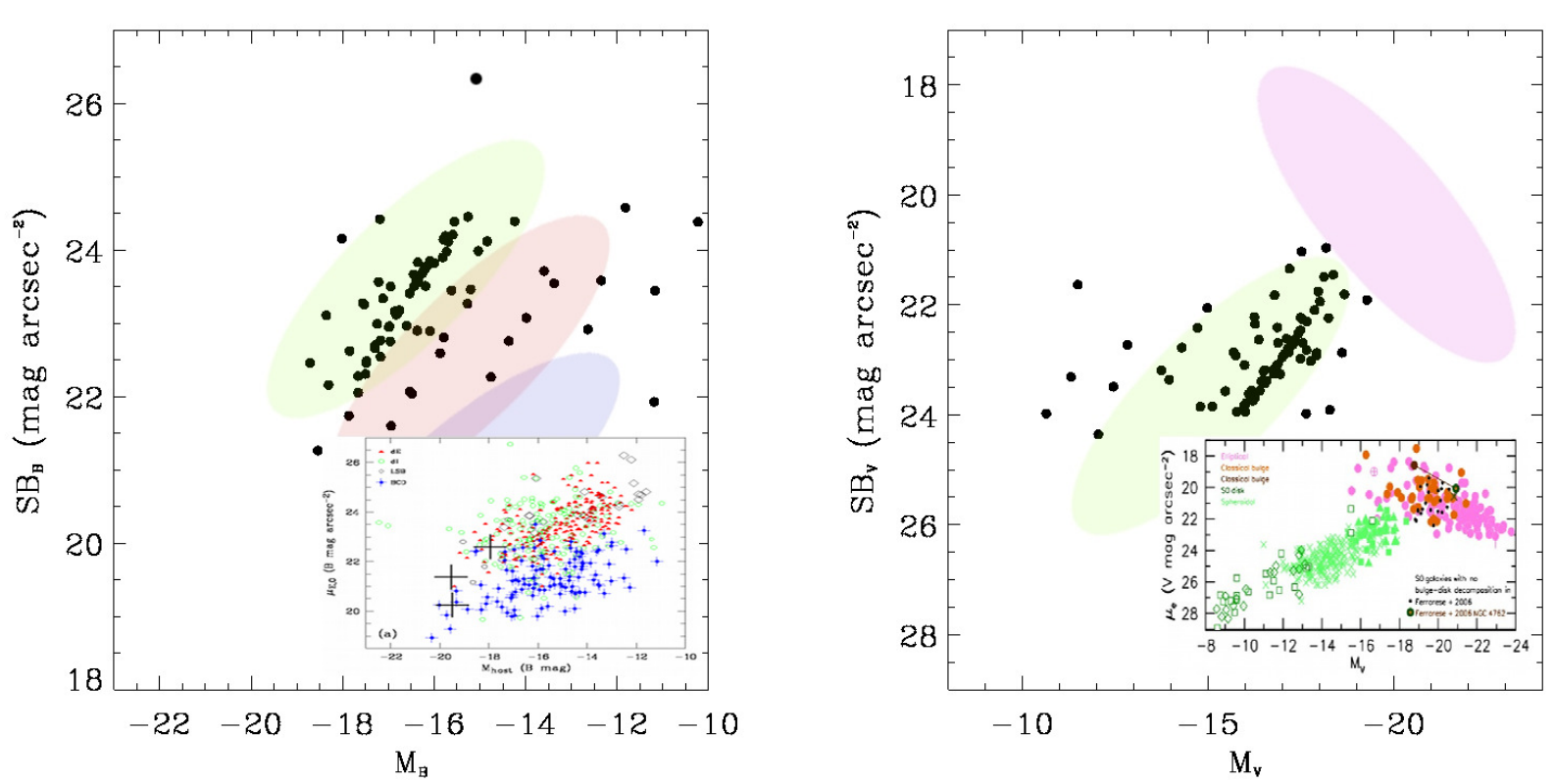

Fig. 23. Surface brightness versus absolute magnitude in the $B$ band (left panel) and $V$ band (right panel) for Sknot galaxies. Figures are adapted from Amorín et al. (2012, their Fig. 9) for $B$ band and Kormendy \& Bender (2012, their Fig. 7) for $V$ band (the inset figure in each panel). Elliptical regions of different colors are overplotted to show the different class of galaxies, following the Amorín et al. (2012) and Kormendy \& Bender (2012) diagrams.

higher values than those of clumps in local spirals, and lower than those found in high-redshift massive star-forming regions.

The $L(\mathrm{H} \alpha)$ versus size of star-forming regions have been investigated by several authors who were searching for a universal relation. Taking the benefit of the excellent spatial resolution provided by the HST images, we have built the $L(\mathrm{H} \alpha)$ versus size for all individual knots of the 220 galaxies of our catalogue. We obtain a slope of $2.48 \pm 0.05$. This is a value similar to 2.5 obtained by Fuentes-Masip et al. (2000) for local, resolved giant HII regions that were measured with the FabryPerot technique. We note, however, that this value differs from the 2.78 slope given by Wisnioski et al. (2012b), in which the authors include high-redshift galaxies. The spatial resolution in Fuentes-Masip et al. (2000) and this work enables us to have a good level of confidence in the results. For high-redshift galaxies the resolution is worse, and can include errors in the measurements, which cannot be quantified.

Sknot galaxies $(\sim 38 \%)$ show photometric structural properties that differ from star-forming knots in the other classes of galaxies (Sknot+diffuse and Mknots). Sknot galaxies have lower surface-brightness and lie in the dwarf spheroidal, dwarf irregular and elliptical regions in the surface-brightness versus absolute magnitude $\left(M_{B}\right.$ and $\left.M_{V}\right)$ diagrams. The possibility of an evolutionary trend among different dwarf systems has already been proposed by Papaderos et al. (1996), and we suggest that Sknot galaxies in COSMOS may be examples of a transitional phase between BCD starbursts and dwarf spheroidals.

Acknowledgements. This work has been funded by the Spanish MINECO, Grant ESTALLIDOS, AYA2013-47742-C4-2P and AYA2010-21887-C04-04. J.M.A. acknowledges support from the European Research Council Starting Grant SEDMorph (P.I. V. Wild). R.H.G. acknowledges the FPI grant from MINECO within ESTALLIDOS project. We thank the referee for his/her constructive comments, which helped to improve the paper. Thanks to Ismael Martínez Delgado for his help with the installation and use of FOCAS software. Thanks to Mercedes Filho for her comments and corrections to the text.

\section{References}

Abraham, R. G., Tanvir, N. R., Santiago, B. X., et al. 1996, MNRAS, 279, L47

Alongi, M., Bertelli, G., Bressan, A., \& Chiosi, C. 1991, A\&A, 244, 95 Amorín, R., Pérez-Montero, E., Vílchez, J. M., \& Papaderos, P. 2012, ApJ, 749, 185

Amorín, R., Sommariva, V., Castellano, M., et al. 2014, A\&A, 568, L8 Aumer, M., Burkert, A., Johansson, P. H., \& Genzel, R. 2010, ApJ, 719, 1230 Bell, E. F., \& de Jong, R. S. 2001, ApJ, 550, 212

Bell, E. F., Phleps, S., Somerville, R. S., et al. 2006, ApJ, 652, 270 Benítez, N., Moles, M., Aguerri, J. A. L., et al. 2009, ApJ, 692, L5 Bertin, E., \& Arnouts, S. 1996, A\&AS, 117, 393

Blanton, M. R., \& Roweis, S. 2007, AJ, 133, 734

Bournaud, F. 2016, in Galactic Bulges (Springer Int. Publ. Switzerland), Astrophys. Space Sci. Libr., 418, 355

Bournaud, F., Elmegreen, B. G., \& Elmegreen, D. M. 2007, ApJ, 670, 237

Bournaud, F., Duc, P.-A., \& Emsellem, E. 2008, MNRAS, 389, L8

Bournaud, F., Elmegreen, B. G., \& Martig, M. 2009, ApJ, 707, L1

Brammer, G. B., van Dokkum, P. G., Franx, M., et al. 2012, ApJS, 200, 13

Bruzual, G., \& Charlot, S. 2003, MNRAS, 344, 1000

Cairós, L. M., Caon, N., García-Lorenzo, B., et al. 2007, ApJ, 669, 251

Cairós, L. M., Caon, N., Papaderos, P., et al. 2009a, ApJ, 707, 1676

Cairós, L. M., Caon, N., Zurita, C., et al. 2009b, A\&A, 507, 1291

Cairós, L. M., Caon, N., Zurita, C., et al. 2010, A\&A, 520, A90

Capak, P., Aussel, H., Ajiki, M., et al. 2007, ApJS, 172, 99

Ceverino, D., Dekel, A., \& Bournaud, F. 2010, MNRAS, 404, 2151

Ceverino, D., Dekel, A., Tweed, D., \& Primack, J. 2015, MNRAS, 447, 3291

Ceverino, D., Sánchez Almeida, J., Muñoz Tuñón, C., et al. 2016, MNRAS, 457, 2605

Chabrier, G. 2003, PASP, 115, 763

Conselice, C. J., Chapman, S. C., \& Windhorst, R. A. 2003, ApJ, 596, L5

Cowie, L. L., Hu, E. M., \& Songaila, A. 1995, AJ, 110, 1576

Dekel, A., Sari, R., \& Ceverino, D. 2009, ApJ, 703, 785

Dekel, A., Zolotov, A., Tweed, D., et al. 2013, MNRAS, 435, 999

Eggen, O. J., Lynden-Bell, D., \& Sandage, A. R. 1962, ApJ, 136, 748

Elmegreen, D. M., Elmegreen, B. G., \& Hirst, A. C. 2004a, ApJ, 604, L21

Elmegreen, D. M., Elmegreen, B. G., \& Sheets, C. M. 2004b, ApJ, 603, 74

Elmegreen, D. M., Elmegreen, B. G., Ravindranath, S., \& Coe, D. A. 2007, ApJ, 658,763

Elmegreen, B. G., Bournaud, F., \& Elmegreen, D. M. 2008, ApJ, 688, 67

Elmegreen, B. G., Elmegreen, D. M., Fernandez, M. X., \& Lemonias, J. J. 2009, ApJ, 692, 12

Elmegreen, B. G., Elmegreen, D. M., Sánchez Almeida, J., et al. 2013, ApJ, 774, 86 
Fuentes-Masip, O., Muñoz-Tuñón, C., Castañeda, H. O., \& Tenorio-Tagle, G. 2000, AJ, 120, 752

Genzel, R., Newman, S., Jones, T., et al. 2011, ApJ, 733, 101

Grogin, N. A., Kocevski, D. D., Faber, S. M., et al. 2011, ApJS, 197, 35

Guo, Q. 2011, in EAS Publ. Ser. 48, eds. M. Koleva, P. Prugniel, \& I. Vauglin, 447

Guo, Y., Giavalisco, M., Ferguson, H. C., Cassata, P., \& Koekemoer, A. M. 2012, ApJ, 757, 120

Guo, Y., Ferguson, H. C., Bell, E. F., et al. 2015, ApJ, 800, 39

Hopkins, P. F., Bundy, K., Croton, D., et al. 2010, ApJ, 715, 202

Ilbert, O., Salvato, M., Capak, P., et al. 2008, in Panoramic Views of Galaxy Formation and Evolution, eds. T. Kodama, T. Yamada, \& K. Aoki, ASP Conf. Ser., 399, 169

Ilbert, O., Capak, P., Salvato, M., et al. 2009, ApJ, 690, 1236

Immeli, A., Samland, M., Gerhard, O., \& Westera, P. 2004a, A\&A, 413, 547

Immeli, A., Samland, M., Westera, P., \& Gerhard, O. 2004b, ApJ, 611, 20

Kennicutt, Jr., R. C. 1998, in The Stellar Initial Mass Function, 38th Herstmonceux Conference, eds. G. Gilmore, \& D. Howell, ASP Conf. Ser. 142,1

Kereš, D., Katz, N., Weinberg, D. H., \& Davé, R. 2005, MNRAS, 363, 2

Kewley, L. J., Dopita, M. A., Sutherland, R. S., Heisler, C. A., \& Trevena, J. 2001, ApJ, 556, 121

Kniazev, A. Y., Pustilnik, S. A., Grebel, E. K., Lee, H., \& Pramskij, A. G. 2004, ApJS, 153, 429

Koekemoer, A. M., Faber, S. M., Ferguson, H. C., et al. 2011, ApJS, 197, 36

Kormendy, J., \& Bender, R. 2012, ApJS, 198, 2

Kormendy, J., \& Kennicutt, Jr., R. C. 2004, ARA\&A, 42, 603

Leitherer, C., Schaerer, D., Goldader, J. D., et al. 1999, ApJS, 123, 3

Leitherer, C., Ortiz Otálvaro, P. A., Bresolin, F., et al. 2010, ApJS, 189, 309

L'Huillier, B., Combes, F., \& Semelin, B. 2012, A\&A, 544, A68

Lilly, S. J., Le Fèvre, O., Renzini, A., et al. 2007, ApJS, 172, 70
Lotz, J. M., Madau, P., Giavalisco, M., Primack, J., \& Ferguson, H. C. 2006, ApJ, 636, 592

Mandelker, N., Dekel, A., Ceverino, D., et al. 2014, MNRAS, 443, 3675

Méndez-Abreu, J. 2016, in Galactic Bulges (Switzerland: Springer Int. Publ.), Astrophys. Space Sci. Libr. 418, 15

Miyauchi-Isobe, N., Maehara, H., \& Nakajima, K. 2010, Publications of the National Astronomical Observatory of Japan, 13, 9

Morales-Luis, A. B., Sánchez Almeida, J., Aguerri, J. A. L., \& Muñoz-Tuñón, C. 2011, ApJ, 743, 77

Murata, K. L., Kajisawa, M., Taniguchi, Y., et al. 2014, ApJ, 786, 15

Naab, T., Khochfar, S., \& Burkert, A. 2006, ApJ, 636, L81

Noguchi, M. 1999, ApJ, 514, 77

Overzier, R. A., Heckman, T. M., Tremonti, C., et al. 2009, ApJ, 706, 203

Padilla, N. D., \& Strauss, M. A. 2008, MNRAS, 388, 1321

Papaderos, P., Loose, H.-H., Fricke, K. J., \& Thuan, T. X. 1996, A\&A, 314, 59

Papaderos, P., Guseva, N. G., Izotov, Y. I., \& Fricke, K. J. 2008, A\&A, 491, 113

Puech, M. 2010, MNRAS, 406, 535

Sánchez Almeida, J., Muñoz-Tuñón, C., Elmegreen, D. M., Elmegreen, B. G., \& Méndez-Abreu, J. 2013, ApJ, 767, 74

Sánchez Almeida, J., Elmegreen, B. G., Muñoz-Tuñón, C., \& Elmegreen, D. M. 2014, A\&ARv, 22, 71

Sobral, D., Smail, I., Best, P. N., et al. 2013, MNRAS, 428, 1128

Tadaki, K.-I., Kodama, T., Tanaka, I., et al. 2014, ApJ, 780, 77

van de Voort, F., Schaye, J., Booth, C. M., Haas, M. R., \& Dalla Vecchia, C. 2011, MNRAS, 414, 2458

van den Bergh, S., Abraham, R. G., Ellis, R. S., et al. 1996, AJ, 112, 359

Vázquez, G. A., \& Leitherer, C. 2005, ApJ, 621, 695

Wang, J., Navarro, J. F., Frenk, C. S., et al. 2011, MNRAS, 413, 1373

Whitaker, K. E., van Dokkum, P. G., Brammer, G., \& Franx, M. 2012a, ApJ, 754, L29

Wisnioski, E., Glazebrook, K., Blake, C., et al. 2012b, MNRAS, 422, 3339

Wuyts, S., Förster Schreiber, N. M., Genzel, R., et al. 2012, ApJ, 753, 114 